\title{
CLASSIFICATION OF THE TOR-ALGEBRAS OF CODIMENSION FOUR ALMOST COMPLETE INTERSECTIONS
}

\author{
ANDREW R. KUSTIN
}

\begin{abstract}
Let $(R, \mathrm{~m}, k)$ be a local ring in which 2 is a unit. Assume that every element of $k$ has a square root in $k$. We classify the algebras $\operatorname{Tor}_{0}^{R}(R / J, k)$ as $J$ varies over all grade four almost complete intersection ideals in $R$. The analogous classification has already been found when $J$ varies over all grade four Gorenstein ideals [21], and when $J$ varies over all ideals of grade at most three [5,30]. The present paper makes use of the classification, in [21], of the Tor-algebras of codimension four Gorenstein rings, as well as the (usually nonminimal) DG-algebra resolution of a codimension four almost complete intersection which is produced in [ 25 and 26$]$.
\end{abstract}

Fix, for the time being, a regular local ring $(R, \mathfrak{m}, k)$. For each CohenMacaulay ring $A$ of the form $A=R / I$, we consider the Tor-algebra $T_{0}=$ $T_{0}(A)=\operatorname{Tor}_{0}^{R}(A, k)$. A great deal of information about $A$ is encoded in $T_{0}(A)$. Some of the classical results along these lines are: $A$ is regular if and only if $T_{\bullet}=T_{0}$ [27]; $A$ is Gorenstein if and only if $T_{\bullet}$ is a Poincare duality algebra [4]; $A$ is a complete intersection if and only if $T_{\bullet}$ is the exterior algebra on $T_{1}[29,1]$. There are at least three types of modern applications of theorems which classify Tor-algebras. The major impetus for studying $T_{0}$ is Avramov's machine for converting questions about the local ring $A$ into questions about the algebra $T_{\bullet}$, provided the minimal $R$-free resolution of $A$ is a DG-algebra. The algebra $T_{\bullet}$ is graded-commutative, instead of commutative; nonetheless, it is a much simpler object than the original ring $A$. In particular, $T_{0}$ is always a finite dimensional vector space over $k$. Avramov's machine has been successfully applied when the codimension of $A$ is at most three; or $A$ is Gorenstein of codimension four; or $A$ is one link from a complete intersection; or $A$ is Gorenstein and two links from a complete intersection. In each case the minimal $R$-resolution of $A$ is a DG-algebra $[6,17,19,16,5]$ and the Tor-algebra $T_{\bullet}(A)$ has been classified $[21,30,5]$. Once the key hypotheses are established, then one is able to prove $[12,5]$ that the Poincare series

$$
P_{A}^{M}(z)=\sum_{i=0}^{\infty} \operatorname{dim}_{k} \operatorname{Tor}_{i}^{A}(M, k) z^{i}
$$

is a rational function for all finitely generated $A$-modules $M$. One is also able to prove [2] that all of these rings $A$ satisfy the Eisenbud Conjecture [8]; that

Received by the editors May 1, 1991.

1991 Mathematics Subject Classification. Primary 13D03, 13C40, 14M07.

Key words and phrases. Almost complete intersection, Betti numbers, DG-algebra, Gorenstein ideal, linkage, perfect ideal, Poincaré series, Tor-algebra. 
is, if $M$ is a finitely generated $A$-module whose Betti numbers are bounded, then the minimal resolution of $M$ is eventually periodic of period two. See [3] for further results and problems along these lines.

Avramov's machine has been applied to Gorenstein rings of codimension four and to rings which are a "small" number of links from other "nice" rings. It is our hope that these techniques may also be applied to rings which are one link from a Gorenstein ring of codimension four, in other words to almost complete intersections of codimension four. The first step in this direction was taken in Palmer's thesis [25, 26]. Let $A$ be a codimension four almost complete intersection. Palmer produced a DG-algebra resolution of $A$. Palmer's resolution is close to, but not always equal to, the minimal resolution of $A$. Palmer's work provides evidence that the minimal resolution of $A$ is a DG-algebra and it is very useful in the present paper where the second step-the classification of $T_{0}(A)$-takes place. Palmer's work is summarized in $\S 3$, and is applied to $T_{0}(A)$ in $\S 4$. (It is noteworthy that the present paper represents the first time that $T_{\bullet}(A)$ has been classified before the minimal resolution of $A$ was known to be a DG-algebra; indeed, it is likely that the present work will help complete the project initiated in [25].)

A second application of theorems which classify Tor-algebras is to the Buchsbaum-Eisenbud conjecture [6] about lower bounds for Betti numbers. Charalambous, Evans, and Miller [7] have proved that if the dimension, $d$, of $R$ is at most four, and $M$ is an $R$-module of finite length, with $M$ not equal to $R$ modulo a regular sequence, then the Betti numbers of $M$ satisfy $\left(\begin{array}{l}d \\ i\end{array}\right) \leq \beta_{i}(M)$ for $0<i<d$, and $2^{d}+2^{d-1} \leq \sum_{i=0}^{d} \beta_{i}(M)$. One of the key ingredients in their proof is the classification in [21] of $T_{\bullet}(A)$ for codimension four Gorenstein rings $A$. The classification of Tor-algebras contained in the present paper should lead to further progress on establishing lower bounds for Betti numbers.

Multiplicative operations in Tor-algebras also play some role in determining the generating set of a residual intersection. This theme is initiated in [23]. Further results along these lines will appear in subsequent papers.

The algebra $T_{\bullet}(A)$ has been classified when $A$ is a codimension four Gorenstein ring [21]; and when $A$ is a codimension three ring [30,5]. In each case, there are at most five different families of Tor-algebras. Furthermore, each family is discrete, in the sense that the family members are parameterized by integers. The proofs in [21] and [5] are based on the theory of linkage. The proof in [30] comes from invariant theory. The proofs look quite different, but the ultimate linear algebra calculations are roughly equivalent. The linkage theory proof is like an induction; one must know the answer before one can prove it. For rings of codimenson three, the proof in [30] preceded proof in [5]; indeed, the authors of [5] took Weyman's answer and reproved it using their linkage technique. Some further details may be found in [24]. The classification in the present paper uses the linkage style of argument. Once again the answer consists of a small number of discrete families of Tor-algebras; see Theorem 1.5.

The main result is stated in $\S 1$ and proved in $\S 4$. Palmer's DG-algebra resolution $\mathbb{M}$ of a codimension four almost complete intersection is recorded in $\S 3$. The multiplication in $\mathbb{M}$ uses the multiplication on a resolution of a codi- 
mension four Gorenstein ring. In $\S 2$ we recall the classification of $T_{\bullet}(A)$ for codimension four Gorenstein rings $A$. In $\S 5$ we give examples and ask questions. The remainder of the present section is a discussion of the conventions that are used throughout the paper.

In this paper "ring" means commutative noetherian ring with one. The grade of a proper ideal $I$ in a ring $R$ is the length of the longest regular sequence on $R$ in $I$. The ideal $I$ of $R$ is called perfect if the grade of $I$ is equal to the projective dimension, $\operatorname{pd}_{R}(R / I)$, of the $R$-module $R / I$. A grade $g$ ideal $I$ is called a complete intersection if it can be generated by $g$ generators. Complete intersection ideals are necessarily perfect. The grade $g$ ideal $I$ is called an almost complete intersection if it is a perfect ideal which is not a complete intersection and which can be generated by $g+1$ generators. The grade $g$ ideal $I$ is called Gorenstein if it is perfect and $\operatorname{Ext}_{R}^{g}(R / I, R) \cong R / I$.

Let $k$ be a fixed field. Throughout this paper, we write

$$
\text { " } S_{\bullet} \text { is a graded } k \text {-algebra" }
$$

to mean that $S_{\bullet}$ is a finite dimensional graded-commutative associative $k$ algebra of the form $S_{\bullet}=\bigoplus_{i=0}^{n} S_{i}$ with $S_{0}=k$. In particular,

and

$$
s_{i} s_{j}=(-1)^{i j} s_{j} s_{i} \text { for all } s_{i} \in S_{i} \text { and } s_{j} \in S_{j}
$$

$$
s_{i} s_{i}=0 \text { if } s_{i} \in S_{i} \text { and } i \text { is odd. }
$$

For example, if $(R, \mathfrak{m}, k)$ is a local ring and $I$ is an $R$-ideal of finite projective dimension, then $\operatorname{Tor}_{\bullet}^{R}(R / I, k)$ is a graded $k$-algebra in the sense of $(0.1)$. For a more concrete example, let $V$ be a vector space of dimension $d$ over $k$. The exterior algebra

$$
\Lambda^{\bullet} V=\Lambda_{k}^{\bullet} V=k \oplus V \oplus \Lambda^{2} V \oplus \bigwedge^{3} V \oplus \cdots \oplus \bigwedge^{d} V
$$

with multiplication given by exterior product, is a graded $k$-algebra in the sense of $(0.1)$. We use the usual conventions regarding grading. If $M=\bigoplus M_{j}$ is a graded $S_{0}$-module, then $M(a)$ is the graded $S_{\bullet}$-module with the property that $M(a)_{j}=M_{a+j}$ and $\operatorname{Hom}_{S_{\mathbf{0}}}\left(S_{\bullet}(-a), M\right)=M(a)$. In particular, there is an isomorphism of graded $k$-vector spaces from $k(-1)^{d}$ to the subspace $V=\bigwedge^{1} V$ of $\bigwedge^{\bullet} V$.

In this paper the word "trivial" is given two distinct meanings. Suppose that $S_{\bullet}$ is a graded $k$-algebra and $W$ is a positively graded $S_{\bullet}$-module. Then the trivial extension of $S_{\bullet}$ by $W, S_{\bullet} \ltimes W$, is the graded $k$-algebra whose graded vector space structure is given by $S_{\bullet} \oplus W$ and whose multiplication is given by

$$
\left(s_{i}, w_{j}\right)\left(s_{k}, w_{l}\right)=\left(s_{i} s_{k}, s_{i} w_{l}+(-1)^{j k} s_{k} w_{j}\right)
$$

for all $s_{a} \in S_{a}$ and all $w_{b} \in W_{b}$. On the other hand, we say that $W$ is a trivial $S_{\bullet}$-module if $S_{+} W=0$. In particular, if $k$ is viewed as an $S_{\bullet}$-module by way of the natural quotient map $S_{\bullet} \rightarrow S_{\bullet} / S_{+}=k$, then $\bigoplus_{i=1}^{n} k(-i)^{m_{i}}$ is a trivial S.-module.

Elementary results about linkage and DG-algebras may be found in [6 and 17]. In this paper, "DG-algebra" always means associative DG-algebra.

\section{THE STATEMENT OF THE MAIN THEOREM}

Let $k$ be a fixed field. In Table 1.3 we define the graded $k$-algebras (in the sense of (0.1)) which appear in Theorem 1.5, the main theorem of the 
paper. Each of these algebras has the form $S_{\bullet}=\bigoplus_{i=0}^{4} S_{i}$ with $S_{0}=k$ and $d_{i}=\operatorname{dim}_{k} S_{i}$. Select bases $\left\{x_{i}\right\}$ for $S_{1},\left\{y_{i}\right\}$ for $S_{2},\left\{z_{i}\right\}$ for $S_{3}$, and $\left\{w_{i}\right\}$ for $S_{4}$. View $S_{2}$ as the direct sum $S_{2}^{\prime} \oplus S_{1}^{2}$. Numerical information about these algebras is collected in Table 1.4. One may combine Lemma 1.2 with Table 1.4 in order to conclude that each of the algebras of Table 1.3 represents a distinct isomorphism class of $k$-algebras, provided the parameters $p, q$, and $r$ satisfy

$$
0 \leq p, \quad 2 \leq q \leq 3, \quad \text { and } \quad 2 \leq r \leq 5
$$

(If we had allowed $q$ and $r$ to take the value 1 , then $\mathbf{E}^{(1)}$ would equal $\mathbf{E}[1]$ and $\mathbf{F}^{(1)}$ would equal $\mathbf{F}[1]$.)

Lemma 1.2. If $S_{\bullet}$ is one of the algebras from Table 1.3, then there is a four dimensional subspace $V$ of $S_{1}$ with the property that $\operatorname{dim} V^{2}=6$ if and only if $S_{\bullet}$ is not equal to $\mathbf{C}^{(2)}, \mathbf{C}^{\star}$, or $\mathbf{C}[p]$ for any $p$.

Proof. If $S_{\bullet}$ is not equal to $\mathbf{C}^{(2)}, \mathbf{C}^{\star}$, or $\mathbf{C}[p]$ for any $p$, then the subspace $V$ of $S_{1}$ spanned by $x_{1}, x_{2}, x_{3}$, and $x_{4}$ has $\operatorname{dim} V^{2}=6$. On the other hand, we now suppose that $S_{\bullet}$ is equal to $\mathbf{C}^{(2)}, \mathbf{C}^{\star}$, or $\mathbf{C}[p]$ for some $p$. Let $x_{1}^{\prime}, x_{2}^{\prime}, x_{3}^{\prime}, x_{4}^{\prime}$ be a basis for $V$. Select $\alpha_{i j}$ in $k$ with $x_{j}^{\prime}=\sum_{i=1}^{5} \alpha_{i j} x_{i}$; let $\Delta(i, j ; a, b)$ and $D(a, b, c, d)$ represent the following determinants:

$$
\Delta(i, j ; a, b)=\left|\begin{array}{ll}
\alpha_{i a} & \alpha_{i b} \\
\alpha_{j a} & \alpha_{j b}
\end{array}\right| \quad \text { and } \quad D(a, b, c, d)=\left|\begin{array}{llll}
\alpha_{a 1} & \alpha_{a 2} & \alpha_{a 3} & \alpha_{a 4} \\
\alpha_{b 1} & \alpha_{b 2} & \alpha_{b 3} & \alpha_{b 4} \\
\alpha_{c 1} & \alpha_{c 2} & \alpha_{c 3} & \alpha_{c 4} \\
\alpha_{d 1} & \alpha_{d 2} & \alpha_{d 3} & \alpha_{d 4}
\end{array}\right| .
$$

Recall that $x_{3} x_{4}=x_{3} x_{5}=x_{4} x_{5}=0$ in $S_{\bullet}$. It follows that

$$
x_{a}^{\prime} x_{b}^{\prime}=\sum_{i=1}^{2} \sum_{j=i+1}^{5} \Delta(i, j ; a, b) x_{i} x_{j} \quad \text { in } S_{\bullet} .
$$

Observe that

$$
\begin{gathered}
\Delta(1,2 ; 3,4) x_{1}^{\prime} x_{2}^{\prime}-\Delta(1,2 ; 2,4) x_{1}^{\prime} x_{3}^{\prime}+\Delta(1,2 ; 2,3) x_{1}^{\prime} x_{4}^{\prime}+\Delta(1,2 ; 1,2) x_{3}^{\prime} x_{4}^{\prime} \\
-\Delta(1,2 ; 1,3) x_{2}^{\prime} x_{4}^{\prime}+\Delta(1,2 ; 1,4) x_{2}^{\prime} x_{3}^{\prime}=\sum_{i=1}^{2} \sum_{j=i+1}^{5} D(1,2, i, j) x_{i} x_{j}=0 .
\end{gathered}
$$

There are two possibilities. If $\Delta(1,2 ; a, b) \neq 0$ for some pair $(a, b)$, then $\operatorname{dim} V^{2} \leq 5$. If $\Delta(1,2 ; a, b)=0$ for all $(a, b)$, then the rank of

$$
\left[\begin{array}{llll}
\alpha_{11} & \alpha_{12} & \alpha_{13} & \alpha_{14} \\
\alpha_{21} & \alpha_{22} & \alpha_{23} & \alpha_{24}
\end{array}\right]
$$

is at most one, and $V$ is contained in $U=\left(\lambda x_{1}+\mu x_{2}, x_{3}, x_{4}, x_{5}\right)$ for some $\lambda$ and $\mu$ in $k$. It follows that $\operatorname{dim} V^{2} \leq \operatorname{dim} U^{2} \leq 3$.

Key to Table 1.3.

(a) $x_{1} x_{2}=y_{1}, x_{1} x_{3}=y_{2}, x_{1} x_{4}=y_{3}, x_{2} x_{3}=y_{4}, x_{2} x_{4}=y_{5}, x_{3} x_{4}=y_{6}$

(a') $x_{1} x_{2} x_{3}=z_{1}, x_{1} x_{2} x_{4}=z_{2}, x_{1} x_{3} x_{4}=z_{3}, x_{2} x_{3} x_{4}=z_{4}$

(b) $x_{1} x_{2}=y_{l+1}, x_{1} x_{3}=y_{l+2}, x_{1} x_{4}=y_{l+3}, x_{1} x_{5}=y_{l+4}, x_{2} x_{3}=y_{l+5}$, $x_{2} x_{4}=y_{l+6}, x_{3} x_{4}=y_{l+7}$

(b') $x_{1} x_{2} x_{3}=z_{l+1}, x_{1} x_{2} x_{4}=z_{l+2}, x_{1} x_{3} x_{4}=z_{l+3}$

(c) $x_{1} x_{2}=y_{l+1}, x_{1} x_{3}=y_{l+2}, x_{1} x_{4}=y_{l+3}, x_{1} x_{5}=y_{l+4}, x_{2} x_{3}=y_{l+5}$, $x_{2} x_{4}=y_{l+6}, x_{2} x_{5}=y_{l+7}$ 


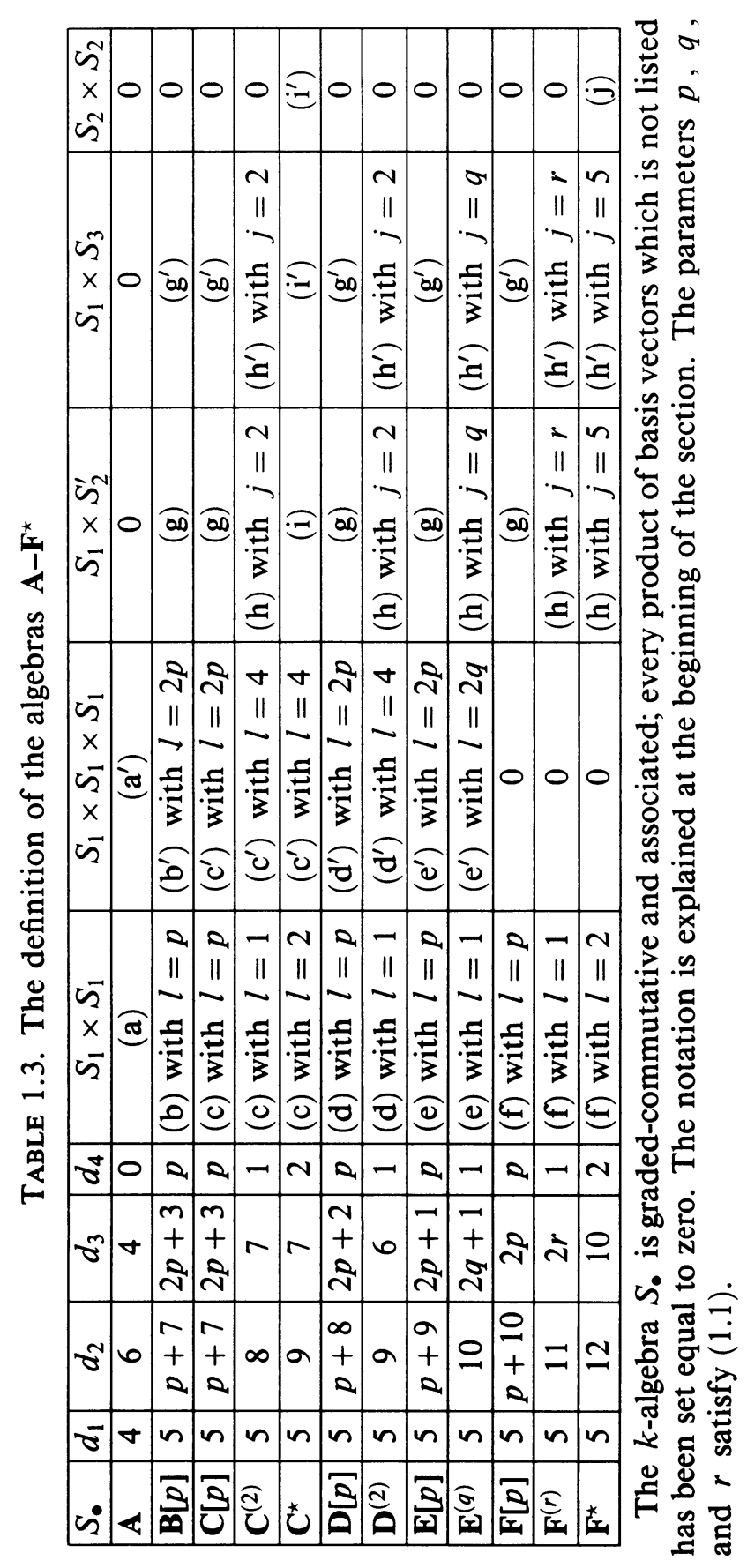


(c') $x_{1} x_{2} x_{3}=z_{l+1}, x_{1} x_{2} x_{4}=z_{l+2}, x_{1} x_{2} x_{5}=z_{l+3}$

(d) $x_{1} x_{2}=y_{l+1}, x_{1} x_{3}=y_{l+2}, x_{1} x_{4}=y_{l+3}, x_{1} x_{5}=y_{l+4}, x_{2} x_{3}=y_{l+5}$, $x_{2} x_{4}=y_{l+6}, x_{2} x_{5}=y_{l+7}, x_{3} x_{4}=y_{l+8}$

(d') $x_{1} x_{2} x_{3}=z_{l+1}, x_{1} x_{2} x_{4}=z_{l+2}$

(e) $x_{1} x_{2}=y_{l+1}, x_{1} x_{3}=y_{l+2}, x_{1} x_{4}=y_{l+3}, x_{1} x_{5}=y_{l+4}, x_{2} x_{3}=y_{l+5}$, $x_{2} x_{4}=y_{l+6}, x_{2} x_{5}=y_{l+7}, x_{3} x_{4}=y_{l+8}, x_{3} x_{5}=y_{l+9}$

(e') $x_{1} x_{2} x_{3}=z_{l+1}$

(f) $x_{1} x_{2}=y_{l+1}, x_{1} x_{3}=y_{l+2}, x_{1} x_{4}=y_{l+3}, x_{1} x_{5}=y_{l+4}, x_{2} x_{3}=y_{l+5}$, $x_{2} x_{4}=y_{l+6}, x_{2} x_{5}=y_{l+7}, x_{3} x_{4}=y_{l+8}, x_{3} x_{5}=y_{l+9}, x_{4} x_{5}=y_{l+10}$

(g) $x_{1} y_{i}=z_{i}$ for $1 \leq i \leq p$,

(g') $x_{1} z_{p+i}=w_{i}$ for $1 \leq i \leq p$,

(h) $x_{i} y_{1}=z_{i}$ for $1 \leq i \leq j$,

(h') $x_{i} z_{j+i}=w_{1}$ for $1 \leq i \leq j$,

(i) $x_{1} y_{1}=z_{1}, x_{1} y_{2}=z_{2}, x_{2} y_{1}=z_{3}, x_{2} y_{2}=z_{4}$

(i') $x_{1} x_{2} y_{1}=w_{1}, x_{1} x_{2} y_{2}=w_{2}$

(j) $y_{1} y_{2}=w_{1}, y_{1}^{2}=w_{2}$.

TABLE 1.4. Numerical information about the algebra $\mathbf{A}-\mathbf{F}^{\star}$

\begin{tabular}{l|c|c|c|c|c|}
$S_{\bullet}$ & $\operatorname{dim} S_{1}^{2}$ & $\operatorname{dim} S_{1}^{3}$ & $\operatorname{dim} S_{1} S_{2}-\operatorname{dim} S_{1}^{3}$ & $\operatorname{dim} S_{1} S_{3}$ & $\operatorname{dim} S_{2}^{2}$ \\
\hline $\mathbf{A}$ & 6 & 4 & 0 & 0 & 0 \\
\hline $\mathbf{B}[p]$ & 7 & 3 & $p$ & $p$ & 0 \\
\hline $\mathbf{C}[p]$ & 7 & 3 & $p$ & $p$ & 0 \\
\hline $\mathbf{C}^{(2)}$ & 7 & 3 & 2 & 1 & 0 \\
\hline $\mathbf{C}^{\star}$ & 7 & 3 & 4 & 2 & 2 \\
\hline $\mathbf{D}[p]$ & 8 & 2 & $p$ & $p$ & 0 \\
\hline $\mathbf{D}^{(2)}$ & 8 & 2 & 2 & 1 & 0 \\
\hline $\mathbf{E}[p]$ & 9 & 1 & $p$ & $p$ & 0 \\
\hline $\mathbf{E}^{(q)}$ & 9 & 1 & $q$ & 1 & 0 \\
\hline $\mathbf{F}[p]$ & 10 & 0 & $p$ & $p$ & 0 \\
\hline $\mathbf{F}^{(r)}$ & 10 & 0 & $r$ & 1 & 0 \\
\hline $\mathbf{F}^{\star}$ & 10 & 0 & 5 & 1 & 2 \\
\hline & & & & &
\end{tabular}

Theorem 1.5. Let $(R, \mathfrak{m}, k)$ be a local ring in which 2 is a unit. Assume that every element of $k$ has a square root in $k$. Let $J$ be a grade four almost complete intersection ideal in $R$, and let $T_{0}$ be the graded $k$-algebra $\operatorname{Tor}_{0}^{R}(R / J, k)$. Then there is a parameter $p, q$, or $r$ which satisfies (1.1), an algebra $S_{0}$ from the list $\mathbf{A}, \mathbf{B}[p], \mathbf{C}[p], \mathbf{C}^{(2)}, \mathbf{C}^{\star}, \mathbf{D}[p], \mathbf{D}^{(2)}, \mathbf{E}[p], \mathbf{E}^{(q)}, \mathbf{F}[p], \mathbf{F}^{(r)}, \mathbf{F}^{\star}$, and a positively graded vector space $W$ such that, $T_{0}$ is isomorphic (as a graded $k$-algebra) to the trivial extension $S_{\bullet} \ltimes W$ of $S_{\bullet}$ by the trivial $S_{\bullet}$-module $W$.

Note. In the above theorem, the vector space $W$ has the form $\bigoplus_{i=1}^{4} k(-i)^{m_{i}}$ where $m_{1}=1$ if $S_{\mathbf{\bullet}}=\mathbf{A}$, and $m_{1}=0$ in all other cases.

The proof of Theorem 1.5 is contained in $\S 4$. We next record a few consequences of Theorem 1.5. If one is interested only in the subalgebra of $T_{\text {. }}$ which is generated by $T_{1}$, then the classification of Theorem 1.5 can be made even cleaner. 
Corollary 1.6. If the notation of Theorem 1.5 is adopted, then the subalgebra $k\left[T_{1}\right]$ of $T_{\bullet}$ is isomorphic to one of the algebras $\mathbf{A} \times k(-1), \mathbf{B}[0], \mathbf{C}[0], \mathbf{D}[0]$, $\mathbf{E}[0], \mathbf{F}[0]$. In particular, the following numerical statements hold:

(a) $6 \leq \operatorname{dim} T_{1}^{2} \leq 10$, and

(b) $\operatorname{dim} T_{1}^{2}+\operatorname{dim} T_{1}^{3}=10$.

Proof. It is easy to see that if $T_{\bullet}$ has the form $S_{\bullet} \times W$ (as described in Theorem 1.5) where $S_{\bullet}$ is $\mathbf{C}^{(2)}$, or $\mathbf{C}^{\star}$, or $\mathbf{C}[p]$ (for some $p$ ), then the subalgebra $k\left[T_{1}\right]$ of $T_{\bullet}$ is $\mathrm{C}[0]$. An analogous statement holds for all of the other algebras of Table 1.3. The numerical assertions follow from Table 1.4.

The next corollary follows from Lemma 1.2 by way of a prime avoidance argument.

Corollary 1.7. Adopt the notation of Theorem 1.5. Exactly one of the following statements holds:

(a) the subalgebra $k\left[T_{1}\right]$ of $T_{\boldsymbol{0}}$ is $\mathbf{C}[0]$; or

(b) there is a minimal presentation

$$
R^{n} \stackrel{d_{2}}{\rightarrow} R^{5} \stackrel{\left[a_{1}, \ldots, a_{5}\right]}{\rightarrow} \mathrm{J}
$$

for $J$ with the property that $a_{1}, a_{2}, a_{3}, a_{4}$ is a regular $R$-sequence and the first six columns of $d_{2}$ are

$$
\left[\begin{array}{cccccc}
-a_{2} & -a_{3} & -a_{4} & 0 & 0 & 0 \\
a_{1} & 0 & 0 & -a_{3} & -a_{4} & 0 \\
0 & a_{1} & 0 & a_{2} & 0 & -a_{4} \\
0 & 0 & a_{1} & 0 & a_{2} & a_{3} \\
0 & 0 & 0 & 0 & 0 & 0
\end{array}\right] .
$$

Remarks 1.9. Some of the algebras of Table 1.3 have a compact coordinate-free representation:

(a) If $V$ is the graded vector space $k(-1)^{4}$, then $\mathbf{A} \cong \Lambda^{\bullet} V / \Lambda^{4} V$. In the notation of Theorem 1.5, one can show (see, for example, [25, Proposition 3.2] or [26, Proposition 4.2]) that $T_{\bullet} \cong \mathbf{A} \ltimes W$ if and only if there is a grade four Gorenstein ideal $I$ and a grade four complete intersection ideal $K$ with

$$
K \subseteq \mathfrak{m} I
$$

such that $J=K: I$. (The significant hypothesis in the last sentence is the one we have isolated as (1.10).)

(b) If $V$ is the graded vector space $k(-1)^{3}$, then $\mathbf{B}[p]$ is isomorphic to

$$
\left(\frac{\Lambda^{\bullet} V}{\bigwedge^{3} V} \ltimes\left[k(-1) \oplus k(-2)^{p} \oplus k(-3)^{p}\right]\right) \otimes_{k} \Lambda^{\bullet} k(-1) .
$$

(c) The algebra $\mathbf{C}[p]$ is isomorphic to

$$
\left[\left[\left(k \ltimes k(-1)^{3}\right) \otimes_{k} \Lambda^{\bullet} k(-1)\right] \ltimes\left(k(-2)^{p} \oplus k(-3)^{p}\right)\right] \otimes_{k} \Lambda^{\bullet} k(-1) .
$$

The algebra $\mathbf{C}^{\star}$ is isomorphic to

$$
\left[k \ltimes\left(k(-1)^{3} \oplus k(-2)^{2}\right)\right] \otimes_{k} \Lambda^{\bullet} k(-1)^{2} .
$$

If $J^{\prime}$ is a grade two almost complete intersection (in other words, $J^{\prime}$ is a determinantal ideal generated by the $2 \times 2$ minors of a $3 \times 2$ matrix), and $J$ 
is the ideal $\left(J^{\prime}, a, b\right)$ for some elements $a$ and $b$ of $R$, with $a, b$ a regular sequence on $R / J^{\prime}$, then $\operatorname{Tor}_{\bullet}^{R}(R / J, k)$ is isomorphic to $\mathbf{C}^{\star}$. (See the proof of case one in $\S 4$.)

(d) Let $V=k(-1)^{2}$ and $V^{\prime}=k(-1)^{2}$ be graded vector spaces and $S_{0}$ be the graded $k$-algebra $\Lambda^{\bullet}\left(V \oplus V^{\prime}\right)$. Let $\overline{S_{\bullet}}$ be the graded $k$-algebra and $W$ be the $\overline{S_{0}}$-module defined by

$$
\overline{S_{\bullet}}=S_{\bullet} /\left(\bigwedge^{2} V^{\prime}\right) S_{+} \quad \text { and } W=S_{\bullet} /\left(V^{\prime}+S_{2}\right) S_{\bullet} .
$$

If $W^{*}$ is the $\overline{S_{\bullet}}$-module $\operatorname{Hom}_{k}(W, k)$, then

$$
\begin{aligned}
\mathbf{D}[0] & =\overline{S_{\bullet}} \ltimes W(-1), \quad \text { and } \\
\mathbf{D}^{(2)} & =\overline{S_{\bullet}} \ltimes\left(W(-1) \oplus W(-2) \oplus W^{*}(-4)\right) .
\end{aligned}
$$

(e) Let $V$ be the graded vector space $k(-1)^{3}, W$ be the $\Lambda^{\bullet} V$-module $\Lambda^{\bullet} V / \Lambda^{2} V$, and $W^{*}$ be the $\Lambda^{\bullet} V$-module $\operatorname{Hom}_{k}(W, k)$. It is not difficult to see that

$$
\begin{aligned}
\mathbf{E}[0] & =\Lambda^{\bullet} V \ltimes W(-1)^{2}, \\
\mathbf{E}^{(3)} & =\Lambda^{\bullet} V \ltimes\left(W(-1)^{2} \oplus W(-2) \oplus W^{*}(-4)\right) .
\end{aligned}
$$

(f) If $V$ is the graded vector space $k(-1)^{5}$, then $\mathbf{F}[0] \cong \Lambda^{\bullet} V / \Lambda^{3} V$. Suppose that $J$ is an ideal from Theorem 1.5 with the property that the subalgebra $k\left[T_{1}\right]$ of $T_{\bullet}$ is isomorphic to $\mathbf{F}[0]$. Let (1.8) be a minimal presentation of $J$. It follows that the basis for $R^{n}$ can be chosen so that the first 10 columns of $d_{2}$ are

$$
\left[\begin{array}{cccccccccc}
-a_{2} & -a_{3} & -a_{4} & -a_{5} & 0 & 0 & 0 & 0 & 0 & 0 \\
a_{1} & 0 & 0 & 0 & -a_{3} & -a_{4} & -a_{5} & 0 & 0 & 0 \\
0 & a_{1} & 0 & 0 & a_{2} & 0 & 0 & -a_{4} & -a_{5} & 0 \\
0 & 0 & a_{1} & 0 & 0 & a_{2} & 0 & a_{3} & 0 & -a_{5} \\
0 & 0 & 0 & a_{1} & 0 & 0 & a_{2} & 0 & a_{3} & a_{4}
\end{array}\right] .
$$

Let $W$ be the $\mathbf{F}[0]$-module $\mathbf{F}[0] / \mathbf{F}_{+}[0]^{2}$, and let $W^{*}$ be the $\mathbf{F}[0]$-module $\operatorname{Hom}_{k}(W, k)$. It is not difficult to see that

$$
\mathbf{F}^{(5)} \cong \mathbf{F}[0] \ltimes\left[W(-2) \oplus W^{*}(-4)\right] .
$$

\section{THE TOR-ALGEBRA OF A CODIMENSION FOUR GORENSTEIN RING}

The classification of Tor-algebras for rings defined by grade four Gorenstein ideals plays a crucial role in the proof of Theorem 1.5. The following result is proved in [21] (when char $k \neq 2$ ) and [16]. (The results in [17, 21], and [16] are stated for Gorenstein ideals in Gorenstein local rings; however, it is not difficult to check that the proofs hold for Gorenstein ideals in arbitrary local rings.) The Tor-algebra $\operatorname{Tor}_{0}^{R}(R / I, k)$ may be described intrinsically without any mention of the minimal resolution

$$
\mathbb{L}: 0 \rightarrow L_{4} \stackrel{l_{4}}{\rightarrow} L_{3} \stackrel{l_{3}}{\rightarrow} L_{2} \stackrel{l_{2}}{\rightarrow} L_{1} \stackrel{l_{1}}{\rightarrow} L_{0}
$$

of $R / I$. We have chosen to introduce $\mathbb{L}$ in Theorem 2.2 so that the notation in the present section coincides with the notation in $\S 4$. We know from [17] and [16] that $\mathbb{L}$ is a DG-algebra; so, the graded $k$-algebras $\overline{\mathbb{L}}$ and $\operatorname{Tor}_{0}^{R}(R / I, k)$ are equal. (Throughout the paper we write ${ }^{-}$to mean $\otimes_{R} k$ and $a \equiv b$ to mean $\bar{a}=\bar{b}$.) 
Theorem 2.2. Let $(R, \mathfrak{m}, k)$ be a local ring. Assume that either every element in $k$ has a square root in $k$, or else that the characteristic of $k$ is equal to two. Let $I$ be a grade four Gorenstein ideal in $R, \mathbb{L}$ be the minimal $R$-resolution of $R / I$, and $\overline{\mathbb{L}}$ be the graded $k$-algebra $\operatorname{Tor}_{\bullet}^{R}(R / I, k)$. If $I$ is not a complete intersection, then there are bases $e_{1}, \ldots, e_{n}$ for $L_{1}$; $f_{1}, \ldots, f_{n-1}, f_{1}^{\prime}, \ldots, f_{n-1}^{\prime}$ for $L_{2} ; g_{1}, \ldots, g_{n}$ for $L_{3} ;$ and $h$ for $L_{4}$ such that the multiplication $\bar{L}_{i} \times \bar{L}_{4-i} \rightarrow \bar{L}_{4}$ is given by

$$
e_{i} g_{j}=\delta_{i j} h, \quad f_{i} f_{j}^{\prime} \equiv \delta_{i j} h, \quad f_{i} f_{j} \equiv f_{i}^{\prime} f_{j}^{\prime} \equiv 0,
$$

and the other products in $\overline{\mathbb{L}}$ are given by one of the following cases:

(a) All products in $\bar{L}_{1} \bar{L}_{1}$ and $\bar{L}_{1} \bar{L}_{2}$ are zero.

(b) All products in $\bar{L}_{1} \bar{L}_{1}$ and $\bar{L}_{1} \bar{L}_{2}$ are zero except:

$$
\begin{gathered}
e_{1} e_{2}=f_{1}, \quad e_{1} e_{3}=f_{2}, \quad e_{2} e_{3}=f_{3}, \\
e_{2} f_{1}^{\prime} \equiv e_{3} f_{2}^{\prime} \equiv g_{1}, \quad-e_{1} f_{1}^{\prime} \equiv e_{3} f_{3}^{\prime} \equiv g_{2}, \quad \text { and } e_{1} f_{2}^{\prime} \equiv e_{2} f_{3}^{\prime} \equiv-g_{3} .
\end{gathered}
$$

(c) There is an integer $p$ such that $e_{p+1} e_{i}=f_{i}, e_{i} f_{i}^{\prime} \equiv g_{p+1}$, and $e_{p+1} f_{i}^{\prime} \equiv$ $-g_{i}$ for $1 \leq i \leq p$ and all other products in $\bar{L}_{1} \bar{L}_{1}$ and $\bar{L}_{1} \bar{L}_{2}$ are zero.

Note. It is possible to choose the basis for $\mathbb{L}$ so that the multiplication is correct "on the nose" for $L_{1} \otimes L_{1} \rightarrow L_{2}$ and $L_{1} \otimes L_{3} \rightarrow L_{4}$, and is also correct for $\bar{L}_{1} \otimes \bar{L}_{2} \rightarrow \bar{L}_{3}$ and $\bar{L}_{2} \otimes \bar{L}_{2} \rightarrow \bar{L}_{4}$.

Remark 2.5. One consequence of the above classification is the well-known fact that $\bar{L}_{1}^{3}=0$ when $I$ is a grade four Gorenstein ideal which is not a complete intersection.

The proof of Theorem 1.5 requires that we understand the multiplication $V \otimes \overline{\mathbb{L}} \rightarrow \overline{\mathbb{L}}$, where $V$ is an arbitrary subspace of $\bar{L}_{1}$. It is not difficult to guess all of the possibilities. For example, if the multiplication of $\overline{\mathbb{L}}$ is described in Theorem 2.2(c), then the distinguished element $\bar{e}_{p+1}$ "may be taken" to be either in $V$ (case (iii) below) or not in $V$ (case (iv)). A complete proof of Corollary 2.7 (in contrast to the above heuristic argument) has two parts. We use linear algebra to find an appropriate basis of $\bar{L}_{1}$, and then we use the fact that $\overline{\mathbb{L}}$ is a Poincare duality algebra to determine the rest of the multiplication in $\overline{\mathbb{L}}$. The second part of the argument is summarized in the following lemma, which appears as [21, Lemma 2.3]. (The characteristic two version of the lemma may be found at the end of [16].) The proof of Lemma 2.6, which is due to Avramov, is the only place in the present paper that the square roots of elements of $k$ are used.

Lemma 2.6. Let $\mathbb{L}$ be as in Theorem 2.2. If $e_{1}, \ldots, e_{n}$ is any basis for $L_{1}$, $h$ is any basis for $L_{4}$, and $f_{1}, \ldots, f_{m}$ is the beginning of a basis for $L_{2}$ with $m \leq n-1$ and $f_{i} f_{j} \equiv 0$ for all $i$ and $j$, then there is a basis $g_{1}, \ldots, g_{n}$ for $L_{3}$ and an extension of $f_{1}, \ldots, f_{m}$ to a basis $f_{1}, \ldots, f_{n-1}, f_{1}^{\prime}, \ldots, f_{n-1}^{\prime}$ for $L_{2}$ such that (2.3) holds.

Corollary 2.7. Adopt the notation and hypotheses of Theorem 2.2. If $V$ is a nonzero subspace of $\bar{L}_{1}$ of dimension $t$, then there are bases $\left\{e_{i}\right\}$ for $L_{1}$, $\left\{f_{i}, f_{i}^{\prime}\right\}$ for $L_{2},\left\{g_{i}\right\}$ for $L_{3}$ and $h$ for $L_{4}$ such that (2.3) holds, $\bar{e}_{1}, \ldots, \bar{e}_{t}$ is a basis for $V$, and the multiplication $V \otimes \bar{L}_{1} \rightarrow \bar{L}_{2}$ and $V \otimes \bar{L}_{2} \rightarrow \bar{L}_{3}$ is given by one of the following cases: 
(i) The integer $t$ is at least 3 and the only nonzero products in $V \bar{L}_{1}$ and $V \bar{L}_{2}$ are given in (2.4).

(ii) The integer $t$ is at least 2 and all products in $V \bar{L}_{1}$ and $V \bar{L}_{2}$ are zero, except

$$
\begin{gathered}
e_{1} e_{2}=f_{1}, \quad e_{1} e_{t+1}=f_{2}, \quad e_{2} e_{t+1}=f_{3}, \\
e_{2} f_{1}^{\prime} \equiv g_{1}, \quad-e_{1} f_{1}^{\prime} \equiv g_{2}, \quad \text { and } \quad e_{1} f_{2}^{\prime} \equiv e_{2} f_{3}^{\prime} \equiv-g_{t+1} .
\end{gathered}
$$

(iii) There are integers $a$ and $b$, with $0 \leq a \leq t-1$ and $0 \leq b$, such that the only nonzero products of basis vectors in $V \bar{L}_{1}$ and $V \bar{L}_{2}$ are

$$
\begin{aligned}
& e_{1} e_{1+i}=f_{i}, \quad e_{1} f_{i}^{\prime} \equiv-g_{1+i}, \quad e_{1+i} f_{i}^{\prime} \equiv g_{1}, \quad \text { for } 1 \leq i \leq a, \\
& e_{1} e_{t+i}=f_{a+i}, \quad e_{1} f_{a+i}^{\prime} \equiv-g_{t+i}, \quad \text { for } 1 \leq i \leq b .
\end{aligned}
$$

(iv) There is an integer $j$, with $2 \leq j \leq t$, such that the only nonzero products of basis vectors in $V \bar{L}_{1}$ and $V \bar{L}_{2}$ are $e_{t+1} e_{i}=f_{i}$ and $e_{i} f_{i}^{\prime} \equiv g_{t+1}$ for $1 \leq i \leq j$. Proof. If $\overline{\mathbb{L}}$ is described in Theorem 2.2(a), then it is clear that $V \overline{\mathbb{L}}$ is given by (iii) with $a=b=0$. We next suppose that $\overline{\mathbb{L}}$ is described by Theorem 2.2(c). In this case $\bar{L}_{1}$ decomposes as $k \bar{e} \oplus U$ for some $e \in L_{1}$ and some $U \subseteq \bar{L}_{1}$ with $U^{2}=0$. There are two possibilities: either $V \subseteq U$, or else there is an element $u$ of $U$ such that $\bar{e}+u \in V$. If $V \subseteq U$, then we let $e_{t+1}$ be the element $e$ of $L_{1}$. Select elements $e_{1}, \ldots, e_{t}$ of $L_{1}$ such that $\bar{e}_{1}, \ldots, \bar{e}_{t}$ is a basis for $V, \bar{e}_{t+1} \bar{e}_{1}, \ldots, \bar{e}_{t+1} \bar{e}_{j}$ is a basis for $\bar{e}_{t+1} V$, and $e_{t+1} e_{i} \equiv 0$ for $j+1 \leq i \leq t$. Define $f_{i}=e_{t+1} e_{i}$ in $L_{2}$ for $1 \leq i \leq j$. Observe that $\left(f_{1}, \ldots, f_{j}\right)^{2}=0$. Complete the basis for $\mathbb{L}$ using Lemma 2.6. Observe that the multiplication $V \overline{\mathbb{L}}$ is described in (iv) (if $2 \leq j$ ) or (iii) (with $a=0$ and $b=j$ if $0 \leq j \leq 1$ ). If $\bar{e}+u \in V$, then let $e_{1} \in L_{1}$ be a preimage of this element. Observe that $\bar{L}_{1}=k \bar{e}_{1} \oplus U$. Select $e_{2}, \ldots, e_{n} \in L_{1}$ such that $\bar{e}_{2}, \ldots, \bar{e}_{n} \in U, \bar{e}_{1} \ldots, \bar{e}_{t}$ is a basis for $V, e_{1}, \ldots, e_{n}$ is a basis for $L_{1}, \bar{e}_{1} \bar{e}_{2}, \ldots, \bar{e}_{1} \bar{e}_{a+1}$ is a basis for $\bar{e}_{1} V, \bar{e}_{1} \bar{e}_{2}, \ldots, \bar{e}_{1} \bar{e}_{a+1}, \bar{e}_{1} \bar{e}_{t+1}, \ldots, \bar{e}_{1} \bar{e}_{t+b}$ is a basis for $\bar{e}_{1} \bar{L}_{1}$, and $e_{1} e_{i} \equiv 0$ whenever $a+2 \leq i \leq t$ or $t+b+1 \leq i \leq n$. Define the elements $f_{1}, \ldots, f_{a+b}$ in $L_{2}$ in the obvious manner and proceed, as in the case $V \subseteq U$, to show that $V \overline{\mathbb{L}}$ is described by (iii).

Finally, suppose that $\overline{\mathbb{L}}$ is described in Theorem 2.2(b). In other words, we are given a decomposition $\bar{L}_{1}=E \oplus U$ with $\operatorname{dim} E=\operatorname{dim} E^{2}=3$ and $U \cdot \bar{L}_{1}=0$. Consider the map $\pi: V \rightarrow E$ which is the composition

$$
V \stackrel{\text { incl. }}{\longrightarrow} \bar{L}_{1}=E \oplus U \stackrel{\text { proj. }}{\longrightarrow} E \text {. }
$$

Let $r$ be the rank of $\pi$. It is clear that the kernel of $\pi$ is $V \cap U$; consequently, we may select $e_{1}, \ldots, e_{t}$ in $L_{1}$ such that $\bar{e}_{1}, \ldots, \bar{e}_{t}$ is a basis for $V$, and $\bar{e}_{r+1}, \ldots, \bar{e}_{t}$ is a basis for $V \cap U$. It follows that $\pi\left(\bar{e}_{1}\right), \ldots, \pi\left(\bar{e}_{r}\right)$ is a basis for $\operatorname{im} \pi$. Let $s=3-r$ and let $e_{t+1}, \ldots, e_{t+s}$ be elements of $L_{1}$ such that $\bar{e}_{t+1}, \ldots, \bar{e}_{t+s}$ are in $E$, and $\pi\left(\bar{e}_{1}\right), \ldots, \pi\left(\bar{e}_{r}\right), \bar{e}_{t+1}, \ldots, \bar{e}_{t+s}$ is a basis for $E$. If $E^{\prime}$ is the subspace $\left(\bar{e}_{1}, \ldots, \bar{e}_{r}, \bar{e}_{t+1}, \ldots, \bar{e}_{t+s}\right)$ of $\bar{L}_{1}$, then it is clear that $\operatorname{dim} E^{\prime}=\operatorname{dim}\left(E^{\prime}\right)^{2}=3$ and that $E^{\prime} \oplus U=\bar{L}_{1}$. It follows that we can find $e_{t+s+1}, \ldots, e_{n}$ in $L_{1}$ such that $\bar{e}_{t+s+1}, \ldots, \bar{e}_{n}$ are in $U$, and $e_{1}, \ldots, e_{n}$ is a basis of $L_{1}$. This basis has been chosen so that $\bar{e}_{1}, \ldots, \bar{e}_{r} \in E^{\prime} \cap V$, $\bar{e}_{r+1}, \ldots, \bar{e}_{t} \in V \cap U, \bar{e}_{t+1}, \ldots, \bar{e}_{t+s} \in E^{\prime} \backslash V, \bar{e}_{t+s+1}, \ldots, \bar{e}_{n} \in U \backslash V$. Complete the basis for $\mathbb{L}$ by using the technique of the preceding paragraph. It is 
now clear that $V \overline{\mathbb{L}}$ is described by (i), if $r=3$; by (ii), if $r=2$; by (iii) with $a=0$ and $b=2$, if $r=1$; and by (iii) with $a=b=0$, if $r=0$.

\section{A DG-RESOLUTION OF ALMOST COMPLETE INTERSECTIONS}

Let $J$ be a grade four almost complete intersection in the local ring $(R, \mathrm{~m}, k)$. In this section we describe Palmer's DG-algebra resolution $\mathbb{M}$ of $R / J$. This resolution, in general, is not the minimal resolution of $R / J$; nonetheless, we are able to use it in $\S 4$ to compute the multiplication in $\operatorname{Tor}_{\bullet}^{R}(R / J, k)$.

Let $K$ be a grade four complete intersection ideal with $K \subseteq J$ and $\mu(J / K)=$ 1. (We use $\mu(M)$ to mean the minimal number of generators of the $R$-module $M$.) The ideal $I=K: J$ is known to be a grade four Gorenstein ideal. It is shown in [17] and [16] (the results in these references hold for Gorenstein ideals in arbitrary local rings) that the minimal resolution $\mathbb{L}$ of $R / I$ is a DG-algebra. Let $\mathbb{K}$ be a Koszul complex which is the minimal resolution of $R / K$ and let $\alpha_{\bullet}: \mathbb{K} \rightarrow \mathbb{L}$

$$
\begin{array}{ccccccccccc}
0 & \rightarrow & K_{4} & \stackrel{k_{4}}{\rightarrow} & K_{3} & \stackrel{k_{3}}{\rightarrow} & K_{2} & \stackrel{k_{2}}{\rightarrow} & K_{1} & \stackrel{k_{1}}{\rightarrow} & K_{0} \\
& \alpha_{4} \downarrow & & \alpha_{3} \downarrow & & \alpha_{2} \downarrow & & \alpha_{1} \downarrow & & \alpha_{0} \downarrow \\
0 & \rightarrow & L_{4} & \stackrel{l_{4}}{\rightarrow} & L_{3} & \stackrel{l_{3}}{\rightarrow} & L_{2} & \stackrel{l_{2}}{\rightarrow} & L_{1} & \stackrel{l_{1}}{\rightarrow} & L_{0}
\end{array}
$$

be a map of DG-algebras which extends the identity map $\alpha_{0}: R \rightarrow R$. Fix orientation isomorphisms [ ]: $K_{4} \rightarrow R$ and [ ]: $L_{4} \rightarrow R$. A routine mapping cone argument establishes the following result.

Proposition 3.2. Let $J$ be a grade four almost complete intersection in the local ring $(R, \mathfrak{m}, k)$ and let $K$ be a grade four complete intersection ideal with $K \subseteq J$ and $\mu(J / K)=1$. Let $\mathbb{K}$ be the minimal resolution of $R / K, \mathbb{L}$ be the minimal resolution of $R / I$ for $I=K: J$, and $\alpha_{\bullet}: \mathbb{K} \rightarrow \mathbb{L}$, as in (3.1), be a map of oriented DG-algebras. If $\beta_{i}: L_{i} \rightarrow K_{i}$ is the map defined by

$$
\left[\beta_{i}\left(v_{i}\right) u_{4-i}\right]=\left[v_{i} \alpha_{4-i}\left(u_{4-i}\right)\right]
$$

for all $u_{j} \in K_{j}$ and all $v_{i} \in L_{i}$, then

$$
\mathbb{M}=\mathbb{M}\left(\alpha_{\bullet}\right): \quad 0 \rightarrow M_{4} \stackrel{m_{4}}{\longrightarrow} M_{3} \stackrel{m_{3}}{\longrightarrow} M_{2} \stackrel{m_{2}}{\longrightarrow} M_{1} \stackrel{m_{1}}{\longrightarrow} M_{0}
$$

is a resolution of $R / J$, where $M_{0}=R, M_{1}=K_{1} \oplus L_{0}, M_{2}=K_{2} \oplus L_{1}$, $M_{3}=K_{3} \oplus L_{2}, M_{4}=L_{3}, m_{1}=\left[\begin{array}{ll}k_{1} & \beta_{0}\end{array}\right]$,

$$
m_{2}=\left[\begin{array}{cc}
k_{2} & -\beta_{1} \\
0 & l_{1}
\end{array}\right], \quad m_{3}=\left[\begin{array}{cc}
k_{3} & \beta_{2} \\
0 & l_{2}
\end{array}\right], \quad \text { and } \quad m_{4}=\left[\begin{array}{c}
-\beta_{3} \\
l_{3}
\end{array}\right] .
$$

Note. The definition of $\beta_{i}$ makes use of the well-known perfect pairings $K_{i} \otimes$ $K_{4-i} \rightarrow R$ and $L_{i} \otimes L_{4-i} \rightarrow R$, which are given by $u_{i} \otimes u_{4-i} \mapsto\left[u_{i} u_{4-i}\right]$ and $v_{i} \otimes v_{4-i} \mapsto\left[v_{i} v_{4-i}\right]$. The orientation on the left side of (3.3) is the orientation on $\mathbb{K}$, whereas the orientation on the right side of $(3.3)$ is the orientation on $\mathbb{L}$.

The next result asserts that $\mathbb{M}$ has the structure of a DG-algebra, provided 2 is a unit in $R$. A small amount of notation is needed in order to describe the multiplication in $\mathbb{M}$. Let $h$ be the element of $L_{4}$ with $[h]=1$ and let $\varepsilon_{1}, \varepsilon_{2}, \varepsilon_{3}, \varepsilon_{4}$ be a basis for $K_{1}$ with $\left[\varepsilon_{1} \wedge \varepsilon_{2} \wedge \varepsilon_{3} \wedge \varepsilon_{4}\right]=1$. The result claims the existence of an $R$-module homomorphism $P: \wedge^{5} L_{1} \rightarrow L_{2}$ which satisfies 
a long list of properties. Two homomorphisms, $p: L_{1} \rightarrow L_{2}$ and $q: L_{2} \rightarrow L_{3}$, are defined in terms of $P$ by

$$
p\left(v_{1}\right)=P\left(v_{1} \wedge \alpha_{1}\left(\varepsilon_{1}\right) \wedge \alpha_{1}\left(\varepsilon_{2}\right) \wedge \alpha_{1}\left(\varepsilon_{3}\right) \wedge \alpha_{1}\left(\varepsilon_{4}\right)\right),
$$

and $v_{1} q\left(v_{2}\right)=v_{2} p\left(v_{1}\right)$ for all $v_{i} \in L_{i}$.

Theorem 3.5 [25]. Adopt the notation of the preceding paragraph together with the notation and hypotheses of Proposition 3.2. If 2 is a unit in $R$, then there is a map $P: \bigwedge^{5} L_{1} \rightarrow L_{2}$ such that the following maps give $\mathbb{M}$ the structure of a DG-algebra:

$$
\begin{aligned}
& M_{1} \otimes M_{1} \rightarrow M_{2}:\left[\begin{array}{l}
u_{1} \\
v_{0}
\end{array}\right]\left[\begin{array}{l}
u_{1}^{\prime} \\
v_{0}^{\prime}
\end{array}\right]= {\left[\begin{array}{c}
u_{1} u_{1}^{\prime} \\
v_{0}^{\prime} \alpha_{1}\left(u_{1}\right)-v_{0} \alpha_{1}\left(u_{1}^{\prime}\right)
\end{array}\right], } \\
& M_{1} \otimes M_{2} \rightarrow M_{3}:\left[\begin{array}{l}
u_{1} \\
v_{0}
\end{array}\right]\left[\begin{array}{c}
u_{2} \\
v_{1}
\end{array}\right]= {\left[\begin{array}{c}
u_{1} u_{2} \\
v_{0} \alpha_{2}\left(u_{2}\right)+\alpha_{1}\left(u_{1}\right) v_{1}+v_{0} p\left(v_{1}\right)
\end{array}\right] } \\
& M_{1} \otimes M_{3} \rightarrow M_{4}:\left[\begin{array}{l}
u_{1} \\
v_{0}
\end{array}\right]\left[\begin{array}{l}
u_{3} \\
v_{2}
\end{array}\right]=-\left[u_{1} u_{3}\right] l_{4}(h)-v_{0} \alpha_{3}\left(u_{3}\right)+\alpha_{1}\left(u_{1}\right) v_{2}-v_{0} q\left(v_{2}\right), \\
& M_{2} \times M_{2} \rightarrow M_{4}:\left[\begin{array}{l}
u_{2} \\
v_{1}
\end{array}\right]\left[\begin{array}{l}
u_{2}^{\prime} \\
v_{1}^{\prime}
\end{array}\right]=-\left[u_{2} u_{2}^{\prime}\right] l_{4}(h)+\alpha_{2}\left(u_{2}\right) v_{1}^{\prime}+v_{1} \alpha_{2}\left(u_{2}^{\prime}\right) \\
&+v_{1} p\left(v_{1}^{\prime}\right)+v_{1}^{\prime} p\left(v_{1}\right)
\end{aligned}
$$

for all $u_{i}, u_{i}^{\prime} \in K_{i}$ and $v_{i}, v_{i}^{\prime} \in L_{i}$. Furthermore, the map $P$ also has the property that

$$
v_{1} v_{1}^{\prime} P\left(v_{1} \wedge v_{1}^{\prime} \wedge{ }_{-}\right): \wedge^{3} L_{1} \rightarrow L_{4}
$$

is the zero map for all $v_{1}, v_{1}^{\prime} \in L_{1}$.

Note. There are two parts to the proof in $[25,26]$. In the first part, a long list of properties for $P$ is compiled such that whenever a map $P$ satisfies all of these properties, then the above indicated multiplication gives $\mathbb{M}$ the structure of a DG-algbra. The one property for $P$ that is highlighted in Theorem 3.5 is just one of the many properties from this list; however, it happens to be the only property of $P$ that we use explicitly in $\S 4$. The second, and much more difficult, part of the proof in $[25,26]$ is to prove that the desired $P$ (a "higher order multiplication" on the resolution $\mathbb{L}$ of a codimension four Gorenstein ring) does exist.

\section{THE PROOF OF THE MAIN THEOREM}

Fix the notation and hypotheses of Theorem 1.5. If $K$ is a grade four complete intersection ideal with $K \subseteq J$ and $\mu(J / K)=1$, then we say that the grade four Gorenstein ideal $I=K: J$ is (directly) linked to $J$ by $K$. For each such $K$, let

$$
t(K)=\operatorname{dim}_{k}\left(\frac{K+\mathfrak{m} I}{\mathfrak{m} I}\right) .
$$

In other words, $t(K)$ is the cardinality of the largest subset of $K$ which begins a minimal generating set for the ideal $K: J$ It is clear that $0 \leq t(K) \leq 4$. Our proof of Theorem 1.5 is divided into three cases:

Case 1. The ideal $J$ is directly linked to a complete intersection. 
Case 2. The ideal $J$ is not directly linked to a complete intersection; and there exists a grade four complete intersection ideal $K$ with $K \subseteq J, \mu(J / K)=1$, and $t(K) \leq 3$.

Case 3. The ideal $J$ is not directly linked to a complete intersection; and $t(K)=4$ for every grade four complete intersection ideal $K$ with $K \subseteq J$ and $\mu(J / K)=1$.

The proof of Theorem 1.5 in Case 1. According to the hypothesis, there are complete intersection ideals $I$ and $K$ with $K \subseteq J, \mu(J / K)=1$, and $I$ linked to $J$ by $K$. Let $t=t(K)$ and $s=4-t$. It is known (see, for example, [5, Theorem 3.2]) that there are matrices $\mathbf{a}_{1 \times s}, \mathbf{b}_{1 \times t}$, and $X_{s \times s}$ with entries in $\mathfrak{m}$ such that $J=J^{\prime}+I_{1}(\mathbf{b})$ and the entries $b_{1}, \ldots, b_{t}$ of $\mathbf{b}$ form a regular sequence on both $R$ and $R / J^{\prime}$ where $J^{\prime}=I_{1}(\mathbf{a} X)+I_{s}(X)$. (If $M$ is a matrix with entries in $R$, then we use $I_{l}(M)$ to denote the ideal in $R$ generated by the $l \times l$ minors of $M$.) Let $\mathbb{L}^{\prime}$ be the minimal resolution of $R / J^{\prime}$ and $\mathbb{K}$ be the Koszul complex which is the minimal resolution of $R / I_{1}(\mathbf{b})$. Both of these resolutions are DG-algebras. (See [5, Proposition 4.4] for the multiplication on $\mathbb{L}^{\prime}$.) It follows that the resolution $\mathbb{L}^{\prime} \otimes_{R} \mathbb{K}$ of $R / J$ is a DG-algebra; and therefore,

$$
T_{\bullet} \cong \operatorname{Tor}_{\bullet}^{R}\left(R / J^{\prime}, k\right) \otimes_{k} \operatorname{Tor}_{\bullet}^{R}\left(R / I_{1}(\mathbf{b}), k\right) .
$$

We know that $\operatorname{Tor}_{\bullet}^{R}\left(R / I_{1}(\mathbf{b}), k\right)$ is the exterior algebra $\bigwedge^{\bullet} k(-1)^{t}$. Proposition 4.4 of [5] shows that

$$
\operatorname{Tor}_{\bullet}^{R}\left(R / J^{\prime}, k\right) \cong S_{\bullet} \ltimes W \quad \text { where } V=k(-1)^{s}, S_{\bullet}=\bigwedge^{\bullet} V / \bigwedge^{s} V,
$$

and $W$ is the trivial $S_{\bullet}$-module

$$
\sum_{i=1}^{s} k(-i)^{\left(\begin{array}{c}
s \\
i-1
\end{array}\right)}
$$

The hypothesis that $J$ is a proper ideal which is not a complete intersection ensures that $0 \leq t \leq 2$. It is now clear that

$$
T_{\bullet}= \begin{cases}\mathbf{C}^{\star}, & \text { if } t=2, \\ \mathbf{B}[3], & \text { if } t=1, \\ \mathbf{A} \ltimes W, & \text { if } t=0 .\end{cases}
$$

The proof of Theorem 1.5 in Case 1 is complete.

For each choice of a grade four complete intersection ideal $K$ with $K \subseteq J$ and $J / K$ cyclic, we are able to use the information of $\S \S 2$ and 3 in order to calculate part of the multiplication in $T_{\bullet}$. To prove Theorem 1.5 in Cases 2 and 3 , we piece together this incomplete information in order to produce the entire multiplication table for $T_{\bullet}$. For the time being, let $K$ be a fixed grade four complete intersection with $K \subseteq J$ and $J / K$ cyclic. Let $t$ denote $t(K)$, and let $I$ be the Gorestein ideal $K: J$. (We are finished with Case 1; so we may assume that the ideal $I$ is not a complete intersection.) Define $\mathbb{K}, \mathbb{L}$, and $\alpha_{\bullet}$ as in $(3.1) ; \beta_{i}$ and $\mathbb{M}=\mathbb{M}\left(\alpha_{\bullet}\right)$ as in Proposition 3.2; and an algebra structure on $\mathbb{M}$ as in Theorem 3.5. We calculate multiplication in $T_{\bullet}$ by using the fact that $T_{\bullet}$ is equal to the homology algebra $H_{\bullet}(\overline{\mathrm{M}})$. A quick look at Proposition 3.2 shows that $T_{\bullet}=\bigoplus_{i=0}^{4} T_{i}$, where $T_{0}=k, T_{1}=\bar{K}_{1} \oplus \bar{L}_{0}$,

$$
T_{2}=\frac{\bar{K}_{2}}{\operatorname{im} \bar{\beta}_{2}} \oplus \bar{L}_{1}, \quad T_{3}=\frac{\bar{K}_{3}}{\operatorname{im} \bar{\beta}_{3}} \oplus \operatorname{ker} \bar{\beta}_{2}, \quad \text { and } \quad T_{4}=\operatorname{ker} \bar{\beta}_{3} .
$$


Much of the multiplication on $\mathbb{M}$ becomes zero in $\bar{M}$. The resolution $\mathbb{L}$ is minimal; and therefore, $l_{4} \equiv 0$. We know from Remark 2.5 that $L_{1}^{3} \subseteq \mathrm{m} L_{3}$; thus,

$$
\operatorname{im} \alpha_{3}=\left(\operatorname{im} \alpha_{1}\right)^{3} \subseteq L_{1}^{3} \subseteq \mathfrak{m} L_{3} \text { and } L_{1} \cdot\left(\operatorname{im} \alpha_{2}\right) \subseteq \mathfrak{m} L_{3} .
$$

It follows that the multiplication of Theorem 3.5 induces the following multiplication on $T_{\bullet}$ :

$$
\begin{aligned}
& T_{1} \otimes T_{1} \rightarrow T_{2}:\left[\begin{array}{c}
\bar{u}_{1} \\
\bar{v}_{0}
\end{array}\right]\left[\begin{array}{l}
\overline{u_{1}^{\prime}} \\
\overline{v_{0}^{\prime}}
\end{array}\right]=\left[\begin{array}{c}
\bar{u}_{1} \bar{u}_{1}^{\prime}\left(\operatorname{modim} \bar{\beta}_{2}\right) \\
\bar{v}_{0}^{\prime} \bar{\alpha}_{1}\left(\bar{u}_{1}\right)-\bar{v}_{0} \bar{\alpha}_{1}\left(\bar{u}_{1}^{\prime}\right)
\end{array}\right], \\
& T_{1} \otimes T_{2} \rightarrow T_{3}:\left[\begin{array}{c}
\bar{u}_{1} \\
\bar{v}_{0}
\end{array}\right]\left[\begin{array}{c}
\bar{u}_{2}\left(\bmod \operatorname{im} \bar{\beta}_{2}\right) \\
\bar{v}_{1}
\end{array}\right]=\left[\begin{array}{c}
\bar{u}_{1} \bar{u}_{2}\left(\operatorname{modim} \bar{\beta}_{3}\right) \\
\bar{v}_{0} \bar{\alpha}_{2}\left(\bar{u}_{2}\right)+\bar{\alpha}_{1}\left(\bar{u}_{1}\right) \bar{v}_{1}+\bar{v}_{0} \bar{p}\left(\bar{v}_{1}\right)
\end{array}\right], \\
& T_{1} \otimes T_{3} \rightarrow T_{4}:\left[\begin{array}{c}
\bar{u}_{1} \\
\bar{v}_{0}
\end{array}\right]\left[\begin{array}{c}
\bar{u}_{3}\left(\bmod \operatorname{im} \bar{\beta}_{3}\right) \\
\left.\bar{v}_{2}\right)
\end{array}\right]=\bar{\alpha}_{1}\left(\bar{u}_{1}\right) \bar{v}_{2}-\bar{v}_{0} \bar{q}_{(}\left(\bar{v}_{2}\right), \\
& T_{2} \otimes T_{2} \rightarrow T_{4}:\left[\begin{array}{c}
\bar{u}_{2}\left(\operatorname{modim} \bar{\beta}_{2}\right) \\
\left.\bar{v}_{1}\right)
\end{array}\right]\left[\begin{array}{c}
\bar{u}_{2}^{\prime}\left(\bmod \operatorname{im} \bar{\beta}_{2}\right) \\
\overline{v_{1}^{\prime}}
\end{array}\right]=\bar{v}_{1} \bar{p}\left(\overline{v_{1}^{\prime}}\right)+\overline{v_{1}^{\prime}} \bar{p}\left(\bar{v}_{1}\right)
\end{aligned}
$$

for $u_{i}, u_{i}^{\prime} \in K_{i}$ and $v_{i}, v_{i}^{\prime} \in L_{i}$.

Apply Corollary 2.7 to the subspace im $\bar{\alpha}_{1}$ of $\bar{L}_{1}$ in the Tor-algebra $\overline{\mathbb{L}}=$ $\operatorname{Tor}_{0}^{R}(R / I, k)$ in order to find bases $e_{1}, \ldots, e_{n}$ for $L_{1} ; f_{1}, \ldots, f_{n-1}, f_{1}^{\prime}, \ldots$, $f_{n-1}^{\prime}$ for $L_{2} ; g_{1}, \ldots, g_{n}$ for $L_{3}$; and $h$ for $L_{4}$ such that $\bar{e}_{1}, \ldots, \bar{e}_{t}$ is a basis for $\operatorname{im} \bar{\alpha}$, and the multiplication $\left(\operatorname{im} \bar{\alpha}_{1}\right) \cdot \overline{\mathbb{L}}$ is described by one of (i)-(iv). In particular, there are five possibilities for the multiplication $\left(\operatorname{im} \bar{\alpha}_{1}\right) \cdot\left(\operatorname{im} \bar{\alpha}_{1}\right)$ :
(A) all products are zero; or
(B) $\bar{e}_{1} \bar{e}_{2}=\bar{f}_{1}$; or
(C) $\bar{e}_{1} \bar{e}_{2}=\bar{f}_{1}$, and $\bar{e}_{1} \bar{e}_{3}=\bar{f}_{2}$; or
(D) $\bar{e}_{1} \bar{e}_{2}=\bar{f}_{1}, \bar{e}_{1} \bar{e}_{3}=\bar{f}_{2}$, and $\bar{e}_{2} \bar{e}_{3}=\bar{f}_{3}$; or
(E) $\bar{e}_{1} \bar{e}_{2}=\bar{f}_{1}, \bar{e}_{1} \bar{e}_{3}=\bar{f}_{2}$, and $\bar{e}_{1} \bar{e}_{4}=\bar{f}_{3}$.

For each possibility we have listed the nonzero products; all other products of basis vectors are zero. In Case 2 of our proof of Theorem 1.5, we have $t \leq 3$, so possibility (E) does not occur in this case. Furthermore, Lemma 4.14(b) shows that in Case 3 the multiplication $\left(\operatorname{im} \bar{\alpha}_{1}\right)^{2}$ is described by (A); consequently there is no loss of generality if we set up our notation under the hypthesis that

$$
\text { the multiplication }\left(\mathrm{im} \bar{\alpha}_{1}\right)^{2} \text { is described by one of (A)-(D). }
$$

Choose a basis $\varepsilon_{1}, \varepsilon_{2}, \varepsilon_{3}, \varepsilon_{4}$ for $K_{1}$ such that

$$
\begin{gathered}
\alpha_{1}\left(\varepsilon_{i}\right)=e_{i} \text { for } 1 \leq i \leq t, \quad \alpha_{1}\left(\varepsilon_{i}\right) \equiv 0 \text { for } t+1 \leq i \leq 4, \quad \text { and } \\
{\left[\varepsilon_{1} \wedge \varepsilon_{2} \wedge \varepsilon_{3} \wedge \varepsilon_{4}\right]=1 .}
\end{gathered}
$$

(Notice that the definition of $p$ in (3.4) appears to use a particular basis for $K_{1}$; however, every basis $\varepsilon_{1}, \varepsilon_{2}, \varepsilon_{3}, \varepsilon_{4}$ of $K_{1}$ which satisfies (4.4) gives rise to the exact same function $p$.) Now that the basis for $K_{1}$ is set, we give names to the corresponding basis elements of $K_{2}$ and $K_{3}$ :

$$
\begin{gathered}
\varphi_{1}=\varepsilon_{1} \varepsilon_{2}, \quad \varphi_{2}=\varepsilon_{1} \varepsilon_{3}, \quad \varphi_{3}=\varepsilon_{2} \varepsilon_{3}, \quad \varphi_{1}^{\prime}=\varepsilon_{3} \varepsilon_{4}, \quad \varphi_{2}^{\prime}=-\varepsilon_{2} \varepsilon_{4}, \quad \varphi_{3}^{\prime}=\varepsilon_{1} \varepsilon_{4}, \\
\gamma_{1}=\varepsilon_{2} \varepsilon_{3} \varepsilon_{4}, \quad \gamma_{2}=-\varepsilon_{1} \varepsilon_{3} \varepsilon_{4}, \quad \gamma_{3}=\varepsilon_{1} \varepsilon_{2} \varepsilon_{4}, \quad \text { and } \gamma_{4}=-\varepsilon_{1} \varepsilon_{2} \varepsilon_{3} .
\end{gathered}
$$


Let $d=\operatorname{rank} \bar{\alpha}_{2}$. It is clear that $0 \leq d \leq\left(\begin{array}{l}t \\ 2\end{array}\right)$. The notation has been set up, thanks to $(4.3)$, so that

$$
\begin{aligned}
\alpha_{2}\left(\varphi_{i}\right)=f_{i} & \text { for } 1 \leq i \leq d, \quad \text { and } \\
\alpha_{2}\left(\varphi_{i}\right) \equiv \alpha_{2}\left(\varphi_{j}^{\prime}\right) \equiv 0 & \text { for } d+1 \leq i \leq 3 \text { and } 1 \leq j \leq 3 .
\end{aligned}
$$

A straightforward application of (3.3) yields

$$
\beta_{3}\left(g_{i}\right) \equiv\left\{\begin{array} { l l } 
{ \gamma _ { i } , } & { \text { if } 1 \leq i \leq t , } \\
{ 0 , } & { \text { if } t + 1 \leq i \leq n , }
\end{array} \quad \beta _ { 2 } ( f _ { i } ^ { \prime } ) \equiv \left\{\begin{array}{ll}
\varphi_{i}^{\prime}, & \text { if } 1 \leq i \leq d, \\
0, & \text { if } d+1 \leq i \leq n-1,
\end{array}\right.\right.
$$

and $\beta_{2}\left(f_{i}\right) \equiv 0$ for $1 \leq i \leq n-1$. Thus,

$$
\begin{aligned}
& \operatorname{ker} \bar{\beta}_{3}=\left(\bar{g}_{t+1}, \ldots, \bar{g}_{n}\right) \subseteq \bar{L}_{3}, \\
& \operatorname{ker} \bar{\beta}_{2}=\left({\overline{f_{d+1}^{\prime}}}_{1}, \ldots,{\overline{f_{n-1}^{\prime}}}, \bar{f}_{1}, \ldots, \bar{f}_{n-1}\right) \subseteq \bar{L}_{3}, \\
& \operatorname{im} \bar{\beta}_{3}=\left(\bar{\gamma}_{1}, \ldots, \bar{\gamma}_{t}\right) \subset \bar{K}_{3} \text {, and } \operatorname{im} \bar{\beta}_{2}=\left(\overline{\varphi_{1}^{\prime}}, \ldots, \overline{\varphi_{d}^{\prime}}\right) \subset \bar{K}_{2} \text {. }
\end{aligned}
$$

Label the following elements of $T_{\bullet}$ :

$$
\begin{gathered}
x_{i}=\left[\begin{array}{c}
\bar{\varepsilon}_{i} \\
0
\end{array}\right], \quad x_{5}=\left[\begin{array}{l}
0 \\
1
\end{array}\right] \in T_{1} \quad \text { for } 1 \leq i \leq 4 ; \\
y_{i}=\left[\begin{array}{c}
\bar{\varphi}_{i}^{\prime}\left(\bmod \operatorname{sim} \bar{\beta}_{2}\right) \\
0
\end{array}\right], \quad y_{3+i}=\left[\begin{array}{c}
\bar{\varphi}_{i}\left(\bmod \operatorname{mim} \bar{\beta}_{2}\right) \\
0
\end{array}\right], \quad y_{6+j}=\left[\begin{array}{c}
0 \\
\bar{e}_{j}
\end{array}\right] \in T_{2}
\end{gathered}
$$

for $1 \leq i \leq 3$ and $1 \leq j \leq n$;

$$
z_{i}=\left[\begin{array}{c}
\bar{\gamma}_{i}\left(\bmod \operatorname{im} \bar{\beta}_{3}\right) \\
0
\end{array}\right], \quad z_{4+j}=\left[\frac{0}{f_{j}}\right], \quad z_{n-d+3+l}=\left[\frac{0}{f_{l}^{\prime}}\right] \in T_{3}
$$

for $1 \leq i \leq 4,1 \leq j \leq n-1$, and $d+1 \leq l \leq n-1$; and

$$
w_{i}=\bar{g}_{t+i} \in T_{4} \text { for } 1 \leq i \leq n-t .
$$

(Notice that the above labeling depends on the choice of $K$.) We see from (4.1) and (4.5) that $y_{1}=\cdots=y_{d}=0, z_{1}=\cdots=z_{t}=0$. Furthermore,

$$
x_{1}, \ldots, x_{5} \text { is a basis for } T_{1} ; y_{d+1}, \ldots, y_{6+n} \text { is a basis for } T_{2} ;
$$

$$
z_{t+1}, \ldots, z_{2 n+2-d}
$$

is a basis for $T_{3}$; and $w_{1}, \ldots, w_{n-t}$ is a basis for $T_{4}$.

It is easy to see, using (4.2), that the multiplication $T_{1} \otimes T_{1} \rightarrow T_{2}$ is given by

$$
\begin{gathered}
x_{1} x_{2}=y_{4}, \quad x_{1} x_{3}=y_{5}, \quad x_{2} x_{3}=y_{6}, \quad x_{3} x_{4}=y_{1}, \quad x_{2} x_{4}=-y_{2}, \\
x_{1} x_{4}=y_{3}, \quad \text { and } \quad x_{i} x_{5}= \begin{cases}y_{6+i}, & \text { for } 1 \leq i \leq t, \\
0, & \text { for } t+1 \leq i \leq 4\end{cases}
\end{gathered}
$$

and that the multiplication $T_{1} \otimes T_{1} \otimes T_{1} \rightarrow T_{3}$ is given by

$$
\begin{aligned}
& x_{2} x_{3} x_{4}=z_{1}, \quad x_{1} x_{3} x_{4}=-z_{2}, \quad x_{1} x_{2} x_{4}=z_{3}, \quad x_{1} x_{2} x_{3}=-z_{4}, \\
& x_{1} x_{2} x_{5}=\left\{\begin{array}{ll}
z_{5}, & \text { if } 1 \leq d, \\
0, & \text { if } d=0,
\end{array} \quad x_{1} x_{3} x_{5}= \begin{cases}z_{6}, & \text { if } 2 \leq d, \\
0, & \text { if } d \leq 1,\end{cases} \right. \\
& x_{2} x_{3} x_{5}= \begin{cases}z_{7}, & \text { if } 3 \leq d, \\
0, & \text { if } d \leq 2,\end{cases}
\end{aligned}
$$


and $x_{i} x_{4} x_{5}=0$ for all $i$. Furthermore, all of the products of basis vectors from

$$
\left(x_{1}, \ldots, x_{4}\right) \cdot\left(y_{t+7}, \ldots, y_{n+6}\right) \text { and }\left(x_{1}, \ldots, x_{4}\right) \cdot T_{3}
$$

are zero except

$$
x_{1} y_{t+7}=z_{6}, \quad x_{2} y_{t+7}=z_{7}, \quad \text { and } \quad x_{1} z_{n+4}=x_{2} z_{n+5}=-w_{1}
$$

when the multiplication $\left(\mathrm{im} \bar{\alpha}_{1}\right) \cdot \overline{\mathbb{L}}$ is described by Corollary 2.7(ii);

$$
x_{1} y_{6+t+i}=z_{4+d+i} \text { and } x_{1} z_{n+3+i}=-w_{i} \quad \text { for } 1 \leq i \leq b
$$

when the multiplication $\left(\mathrm{im} \bar{\alpha}_{1}\right) \cdot \overline{\mathbb{L}}$ is described by Corollary 2.7(iii); and

$$
x_{i} y_{t+7}=-z_{4+i} \text { and } \quad x_{i} z_{n+3+i}=w_{1} \quad \text { for } 1 \leq i \leq j
$$

when the multiplication $\left(\operatorname{im} \bar{\alpha}_{1}\right) \cdot \overline{\mathbb{L}}$ is described by Corollary 2.7 (iv). It is not possible to determine

$$
x_{5} \cdot\left(y_{t+7}, \ldots, y_{n+6}\right), \quad x_{5} \cdot T_{3}, \quad \text { or } T_{2} \cdot T_{2}
$$

at the present level of generality.

The proof of Theorem 1.5 in Case 2. Fix a complete intersection ideal $K$ with $K \subseteq J, \mu(J / K)=1$, and $t(K) \leq 3$. Use $K$ to calculate multiplication in $T_{\bullet}$ as described in (4.1) and (4.2). The map $p$ of (3.4) satisfies $p \equiv 0$ because rank $\bar{\alpha}_{1}=t \leq 3$. The map $q$ is defined in terms of $p$; hence, $q \equiv 0$. It follows that all of the products of (4.12) are zero. Combine the basis for $T_{\text {. }}$ given in (4.6) with the multiplication from (4.7), (4.8), and (4.9) in order to see that Table 4.13 is correct and complete, where $T_{\bullet}=S_{\bullet} \ltimes W$ for some trivial $S_{\bullet}$-module $W$. Recall that the algebras $\mathbf{A}-\mathbf{F}^{\star}$ are defined in Table 1.3. If the multiplication $\left(\operatorname{im} \bar{\alpha}_{1}\right) \cdot \overline{\mathbb{L}}$ is described in part (iii) of Corollary 2.7 , then the parameter $a$ must equal $d$. The multiplications in part (ii) and part (iv) each require that $2 \leq t$; but (ii) must have $d=1$, whereas (iv) requires $d=0$.

TABLE 4.13. The conclusion of the proof of Theorem 1.5 in Case 2

\begin{tabular}{c|c|c|c|c}
$t$ & $d$ & $\left(\mathrm{im} \bar{\alpha}_{1}\right) \cdot \overline{\mathbf{L}}$ & $k\left[T_{1}\right]$ & $S_{\bullet}$ \\
\hline 0 & 0 & $\left(\mathrm{im} \bar{\alpha}_{1}\right)=0$ & $\mathbf{A} \ltimes k(-1)$ & $\mathbf{A}$ \\
\hline 1 & 0 & (iii) with $a=0$ and $b \geq 0$ & $\mathbf{B}[0]$ & $\mathbf{B}[b]$ \\
\hline 2 & 0 & (iii) with $a=0$ and $b \geq 0$ & $\mathbf{D}[0]$ & $\mathbf{D}[b]$ \\
\hline 2 & 0 & (iv) with $j=2$ & $\mathbf{D}[0]$ & $\mathbf{D}^{(2)}$ \\
\hline 2 & 1 & $(\mathrm{ii})$ & $\mathbf{C}[0]$ & $\mathbf{C}^{(2)}$ \\
\hline 2 & 1 & (iii) with $a=1$ and $b \geq 0$ & $\mathbf{C}[0]$ & $\mathbf{C}[b]$ \\
\hline 3 & 0 & (iii) with $a=0$ and $b \geq 0$ & $\mathbf{E}[0]$ & $\mathbf{E}[b]$ \\
\hline 3 & 0 & (iv) with $2 \leq j \leq 3$ & $\mathbf{E}[0]$ & $\mathbf{E}^{(j)}$ \\
\hline 3 & 1 & $(\mathrm{ii})$ & $\mathbf{D}[0]$ & $\mathbf{D}(2)$ \\
\hline 3 & 1 & (iii) with $a=1$ and $b \geq 0$ & $\mathbf{D}[0]$ & $\mathbf{D}[b]$ \\
\hline 3 & 2 & (iii) with $a=2$ and $b \geq 0$ & $\mathbf{B}[0]$ & $\mathbf{B}[b]$ \\
\hline 3 & 3 & $(\mathbf{i})$ & $\mathbf{A} \ltimes k(-1)$ & $\mathbf{A}$ \\
\hline
\end{tabular}


The proof of Theorem 1.5 in Case 2 is complete.

Without any further ado, we are able to identify the subalgebra $k\left[T_{1}\right]$ of $T_{\text {. }}$ in Case 3 of Theorem 1.5. Part (b) of the following lemma appears to be technical; but, in particular, it yields a complete description of the minimal resolution of $R / J$.

Lemma 4.14. If the notation and hyptheses for Case 3 (from the beginning of the present section) are adopted, then the following statements hold:

(a) The algebra $k\left[T_{1}\right]$ is isomorphic to $\mathbf{F}[0]$.

(b) Let $K$ be any grade four complete intersection ideal with $K \subseteq J$ and $\mu(J / K)=1$. If $\mathbb{M}$ from Proposition 3.2 is the corresponding resolution of $R / J$, then $\beta_{2} \equiv 0, \alpha_{2} \equiv 0$, and $\operatorname{im} \beta_{3}=K_{3}$.

Proof. We first prove that $\operatorname{dim}_{k} T_{1}^{2}=10$. Let $\mathbf{a}=\left\{a_{1}, \ldots, a_{5}\right\}$ be a minimal generating set of $J$ with the property that every four element subset of $\mathbf{a}$ is a regular sequence; and let $x_{i}^{\prime}$ be the image of $\bar{a}_{i}$ under the natural isomorphism

$$
J / \mathfrak{m} J \stackrel{\cong}{\rightarrow} \operatorname{Tor}_{1}^{R}(R / J, k) \text {. }
$$

It suffices to show that

$$
\operatorname{dim}_{k}\left(T_{1}^{2} /\left(x_{1}^{\prime}, \ldots, \hat{x}_{i}^{\prime}, \ldots, x_{5}^{\prime}\right)^{2}\right)=4
$$

for $i=1, \ldots, 5$. We establish (4.16) for $i=5$; the other four cases follow from the symmetry of the situation. Let $K$ be the complete intersection ideal $\left(a_{1}, \ldots, a_{4}\right)$. Consider $T_{\bullet}$ as described in (4.1). If $\varepsilon_{1}^{\prime}, \ldots, \varepsilon_{4}^{\prime}$ is a basis for $K_{1}$ with $k_{1}\left(\varepsilon_{i}^{\prime}\right)=a_{i}$, then it follows that

$$
x^{\prime}=\left[\begin{array}{c}
\bar{\varepsilon}_{i}^{\prime} \\
0
\end{array}\right] \quad \text { for } 1 \leq i \leq 4
$$

Let

$$
x_{5}=\left[\frac{0}{1}\right] \text {. }
$$

It is not necessarily true that $x_{5}^{\prime}=x_{5}$; but we do know that $x_{5}^{\prime}=\lambda x_{5}+x^{\prime}$ for some unit $\lambda \in k$ and some $x^{\prime} \in\left(x_{1}^{\prime}, \ldots, x_{4}^{\prime}\right)$. The multiplication in $T_{\bullet}$ can be read from (4.2):

$$
x_{i}^{\prime} x_{j}^{\prime}=\left[\begin{array}{c}
\overline{\varepsilon_{i}^{\prime} \varepsilon_{j}^{\prime}}\left(\operatorname{modim} \bar{\beta}_{2}\right) \\
0
\end{array}\right] \text { and } x_{i}^{\prime} x_{5}=\left[\begin{array}{c}
0 \\
\bar{\alpha}_{1}\left(\overline{\varepsilon_{i}^{\prime}}\right)
\end{array}\right]
$$

for $1 \leq i, j \leq 4$. The hypothesis ensures that $t=4$; so $\alpha_{1}\left(\varepsilon_{1}^{\prime}\right), \ldots, \alpha_{1}\left(\varepsilon_{4}^{\prime}\right)$ is the beginning of a basis for $L_{1}$. We have established that $x_{1}^{\prime} x_{5}^{\prime}, x_{2}^{\prime} x_{5}^{\prime}$, $x_{3}^{\prime} x_{5}^{\prime}$, and $x_{4}^{\prime} x_{5}^{\prime}$ generate a four dimensional subspace of $T_{1}^{2} /\left(x_{1}^{\prime}, \ldots, x_{4}^{\prime}\right)^{2}$; therefore, (4.16) holds and $\operatorname{dim} T_{1}^{2}=10$.

Furthermore, now that we know that $\operatorname{dim} T_{1}^{2}=10$, we may read the preceding paragraph from bottom to top in order to conclude that $\operatorname{im} \bar{\beta}_{2}=0$ for every resolution $\mathbb{M}$ from Proposition 3.2. It is clear that $\operatorname{rank} \bar{\alpha}_{2}=\operatorname{rank} \bar{\beta}_{2}=0$, and that $\operatorname{rank} \bar{\beta}_{3}=\operatorname{rank} \bar{\alpha}_{1}=t=4$; consequently, (b) has been established.

To finish the proof of (a) we must show that $T_{1}^{3}=0$. Once again, we use (4.2) to see that

$$
x_{i}^{\prime} x_{j}^{\prime} x_{l}^{\prime}=\left[\begin{array}{c}
\overline{\varepsilon_{i}^{\prime} \varepsilon_{j}^{\prime} \varepsilon_{l}^{\prime}}\left(\operatorname{modim} \bar{\beta}_{3}\right) \\
0
\end{array}\right] \text { and } x_{i}^{\prime} x_{j}^{\prime} x_{5}=\left[\begin{array}{c}
0 \\
\overline{\alpha_{2}}\left(\overline{\varepsilon_{i}^{\prime} \varepsilon_{j}^{\prime}}\right)
\end{array}\right]
$$


for $1 \leq i, j, l \leq 4$. The product $x_{i}^{\prime} x_{j}^{\prime} x_{l}^{\prime}$ is equal to 0 because $\bar{\beta}_{3}$ is surjective; and $x_{i}^{\prime} x_{j}^{\prime} x_{5}=0$ because $\bar{\alpha}_{2}=0$.

We now subdivide Case 3 into two subcases:

Case 3A. There is a nonzero element $x \in T_{1}$ such that $x T_{2}=0$ and $x T_{3}=0$.

Case 3B. If $x \in T_{1}$ with $x \neq 0$, then either $x T_{2} \neq 0$ or $x T_{3} \neq 0$.

The proof of Theorem 1.5 in Case 3A. Let $a$ be an element of $J$ with the property that $\bar{a}$ is sent to $x$ under the isomorphism of (4.15), and $K$ be a grade four complete intersection ideal such that $J=(K, a)$. Adopt the notation of the paragraph preceding (4.1) and apply Corollary 2.7 in order to pick a basis for $\mathbb{L}$ so that the multiplication in $\left(\operatorname{im} \bar{\alpha}_{1}\right) \cdot \overline{\mathbb{L}}$ is described by one of the cases (i)-(iv). Recall from part (b) of Lemma 4.14 that $\alpha_{2} \equiv 0$; hence, the multiplication $\left(\operatorname{im} \bar{\alpha}_{1}\right) \cdot \overline{\mathbb{L}}$ is actually described by either (iii) with $a=0$ or (iv). Label the elements $x_{i}, y_{i}, z_{i}$, and $w_{i}$ of $T_{\text {. }}$ exactly as was done in (4.6). (Keep in mind that $t=4$ and $d=0$.) Notice that $x_{5}=\lambda x+x^{\prime}$ for some unit $\lambda \in k$ and some $x^{\prime} \in\left(x_{1}, \ldots, x_{4}\right)$. We will know all of the multiplication in

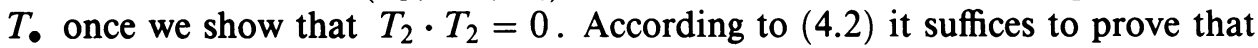
$v_{1}^{\prime} p\left(v_{1}\right) \equiv 0$ for all $v_{1}, v_{1}^{\prime} \in L_{1}$; and therefore, by Remark 2.5 , it suffices to show that $\bar{p}\left(\bar{v}_{1}\right) \in \bar{L}_{1}^{2}$. Since $x^{\prime} \in\left(x_{1}, \ldots, x_{4}\right)$, there is an element $\varepsilon \in K_{1}$ such that

$$
x^{\prime}=\left[\begin{array}{l}
\bar{\varepsilon} \\
0
\end{array}\right] \quad \text { in } T_{1} .
$$

Recall that $x T_{2}=0$. Use (4.2) to compute that

$$
\begin{aligned}
{\left[\begin{array}{c}
0 \\
\bar{\alpha}_{1}\left(\bar{\varepsilon}_{\bar{v}} \bar{v}_{1}\right.
\end{array}\right] } & =\left[\begin{array}{l}
\bar{\varepsilon} \\
0
\end{array}\right]\left[\begin{array}{c}
0 \\
\bar{v}_{1}
\end{array}\right]=x^{\prime}\left[\begin{array}{c}
0 \\
\bar{v}_{1}
\end{array}\right]=x_{5}\left[\begin{array}{c}
0 \\
\bar{v}_{1}
\end{array}\right] \\
& =\left[\begin{array}{l}
0 \\
\overline{1}
\end{array}\right]\left[\begin{array}{c}
0 \\
\bar{v}_{1}
\end{array}\right]=\left[\begin{array}{c}
0 \\
\bar{p}\left(\bar{v}_{1}\right)
\end{array}\right] \in T_{1} \cdot T_{2} .
\end{aligned}
$$

We conclude that $\bar{p}\left(\bar{v}_{1}\right)=\bar{\alpha}_{1}(\bar{\varepsilon}) \bar{v}_{1} \in \bar{L}_{1}^{2}$ and $T_{2}^{2}=0$.

Combine Lemma 4.14(a), together with the hypothesis $x T_{2}=x T_{3}=0$ and the fact $T_{2}^{2}=0$, in order to see that $T_{\bullet}=S_{\bullet} \ltimes W$ for some trivial $S_{\bullet}$-module $W$ where

$$
S_{\bullet}=\left\{\begin{array}{lll}
\mathbf{F}[b] & \text { with } 0 \leq b, & \text { if }(4.9) \text { is described by (4.10), and } \\
\mathbf{F}^{(j)} & \text { with } 2 \leq j \leq 4, & \text { if }(4.9) \text { is described by (4.11). }
\end{array}\right.
$$

The proof of Theorem 1.5 in Case $3 \mathrm{~A}$ is complete.

Case $3 \mathrm{~B}$ is the most interesting case. In Lemma 4.17 we record the consequences in $T_{\bullet}$ of the observation that the multiplication $\left(\operatorname{im} \bar{\alpha}_{1} \cdot \overline{\mathbb{L}}\right)$ must be described by part (iv) of Corollary 2.7. This result gives many incomplete multiplication tables for $T_{0}$. In Lemma 4.18 we paste the incomplete multiplication tables of Lemma 4.17 together to learn all of the multiplication in $T_{\bullet}$, except the multiplication $T_{2}^{2}$. The proof of Lemma 4.20 is where the hard work takes place in Case 3B with $T_{2}^{2} \neq 0$.

Lemma 4.17. Adopt the notation and hypotheses of Case 3B. If $X_{1}$ is a four dimensional subspace of $T_{1}$, then there are elements $y_{1} \in T_{2}$ and $w_{1} \in T_{4}$, and there are subspaces $Y_{1} \subseteq T_{2}, Z_{1} \subseteq T_{3}$, and $Z_{1}^{\prime} \subseteq T_{3}$ such that $T_{2}=k y_{1} \oplus Y_{1}$, $T_{3}=Z_{1} \oplus Z_{1}^{\prime}$, and 
(a) $\operatorname{dim}\left(y_{1} \cdot X_{1}\right)=4$,

(b) $X_{1} \cdot Y_{1}=0$

(c) $X_{1} \cdot T_{3} \subseteq k w_{1}$,

(d) the multiplication map $X_{1} \otimes Z_{1} \rightarrow k w_{1}$ is a perfect pairing,

(e) $X_{1} \cdot Z_{1}^{\prime}=0$, and

(f) $X_{1} \cdot T_{2} \subseteq Z_{1}^{\prime}$.

Proof. Select a grade four complete intersection $K$ with the property that the image of $\bar{K}$ under (4.15) is $X_{1}$. Use $K$ to calculate multiplication in $T_{\bullet}$ as described in (4.1) and (4.2). Observe that the elements $x_{1}, \ldots, x_{4}$, which are defined above (4.6), form a basis for $X_{1}$. We know from Lemma 4.14(a) that $X_{1} \cdot T_{1}^{2}=0$; consequently, all of the multiplication in $X_{1} \cdot T_{2}$ and $X_{1} \cdot T_{3}$ is given in (4.9). Recall the hypothesis that if $x$ is a nonzero element of $X_{1}$, then either $x T_{2} \neq 0$ or $x T_{3} \neq 0$. It follows that the multiplication in $X_{1} \cdot T_{2}$ and $X_{1} \cdot T_{3}$ is described by (4.11) with $j=4$. There is no difficulty seeing that the multiplication of $(4.11)$, with $j=4$, is the same as the coordinate-free description which is given in the statement of the result.

Lemma 4.18. If the notation and hypotheses of Case 3B are adopted, then there are elements $y \in T_{2}$ and $w \in T_{4}$, and there are subspaces $Y \subseteq T_{2}, Z \subseteq T_{3}$, and $Z^{\prime} \subseteq T_{3}$ such that $T_{2}=k y \oplus Y, T_{3}=Z \oplus Z^{\prime}$, and

(a) $\operatorname{dim}\left(y \cdot T_{1}\right)=5$,

(b) $T_{1} \cdot Y=0$,

(c) $T_{1} \cdot T_{3} \subseteq k w$,

(d) the multiplication map $T_{1} \otimes Z \rightarrow k w$ is a perfect pairing,

(e) $T_{1} \cdot Z^{\prime}=0$, and

(f) $T_{1} \cdot T_{2} \subseteq Z^{\prime}$.

Before proving the above result, we notice that Lemmas 4.14 and 4.18 complete the proof in Case $3 \mathrm{~B}$ when $T_{2}^{2}=0$.

Corollary 4.19. If the notation and hypotheses of Case 3B are adopted and $T_{2}^{2}=$ 0 , then $T_{\bullet}$ has the form $\mathbf{F}^{(5)} \ltimes W$ for some trivial $\mathbf{F}^{(5)}$-module $W$.

Proof of Lemma 4.18. Let $X_{1}$ and $X_{2}$ be four dimensional subspaces of $T_{1}$ with $X_{1}=X_{2}$. Apply Lemma 4.17 to find $y_{i} \in T_{2}, w_{i} \in T_{4}, Y_{i} \subseteq T_{2}$, $Z_{i} \subseteq T_{3}$, and $Z_{1}^{\prime} \subseteq T_{3}$ with $\operatorname{dim}\left(y_{i} \cdot X_{i}\right)=4, X_{i} \cdot Y_{i}=0, X_{i} \cdot T_{3} \subseteq k w_{i}$, the multiplication map $X_{i} \otimes Z_{i} \rightarrow k w_{i}$ a perfect pairing, and $X_{i} \cdot Z_{i}^{\prime}=0$, for $i=1$ and $i=2$. Let $y=y_{1}, w=w_{1}$, and $Y=Y_{1}$.

(b) Let $x$ be a nonzero element of $X_{1} \cap X_{2}$ and let $(x)^{\perp}=\left\{y_{0} \in T_{2} \mid x y_{0}=0\right\}$. It is clear that $Y_{1}=(x)^{\perp}=Y_{2}$. Furthermore, we know that $X_{1}+X_{2}=T_{1}$; therefore, $Y \cdot T_{1}=0$.

(a) It suffices to show that $\operatorname{dim}(y \cdot X)=4$ for every four dimensional subspace $X$ of $T_{1}$. The choice of $X_{2}$ is independent of our definition of $y$; consequently, it suffices to show that $\operatorname{dim}\left(y \cdot X_{2}\right)=4$. But, this fact follows from the following observations which we have already established: $k y \oplus Y=k y_{2} \oplus Y$, $\operatorname{dim}\left(y_{2} \cdot X_{2}\right)=4$ and $Y \cdot X_{2}=0$.

(c) Take $x$ from the proof of (b). The hypothesis ensures that $x \cdot T_{3}$ is a nonzero subspace of $\left(w_{1}\right) \cap\left(w_{2}\right)$. It follows that the one dimensional subspaces $\left(w_{1}\right)$ and $\left(w_{2}\right)$ of $T_{4}$ are equal. Use $X_{1}+X_{2}=T_{1}$ in order to conclude that $T_{1} \cdot T_{3} \subseteq(w)$. 
(d) and (e) Let $\varphi$ be the name of the map $T_{3} \rightarrow \operatorname{Hom}_{k}\left(T_{1}, k w\right)$ which is induced by the multiplication map $T_{1} \otimes T_{3} \rightarrow k w$, let $x_{1}, \ldots, x_{5}$ be a fixed basis for $T_{1}$ and let $x_{1}^{*}, \ldots, x_{5}^{*}$ be the corresponding dual basis for $\operatorname{Hom}_{k}\left(T_{1}, k w\right)$. Apply parts (d) and (e) of Lemma 4.17 to the subspace $\left(x_{1}, \ldots, x_{4}\right)$ of $T_{1}$ in order to find a basis for $T_{3}$ for which the matrix of $\varphi$ is

$$
\left[\begin{array}{c|c}
I & 0 \\
\hline \lambda_{1} \cdots \lambda_{4} & \lambda_{5} \cdots \lambda_{n}
\end{array}\right]
$$

for some $\lambda_{i} \in k$. If $\lambda_{5}=\cdots=\lambda_{n}=0$, then $x \cdot T_{3}=0$ for $x=x_{5}-\sum_{i=1}^{4} \lambda_{i} x_{i}$ and this contradicts Lemma 4.17(d). Thus, $\lambda_{i} \neq 0$ for some $i$ with $5 \leq i \leq n$ and a basis $z_{1}, \ldots, z_{n}$ for $T_{3}$ may be found for which the matrix of $\varphi$ is $\left[\begin{array}{ll}I & 0\end{array}\right]$. Let $Z=\left(z_{1}, \ldots, z_{5}\right)$ and $Z^{\prime}=\left(z_{6}, \ldots, z_{n}\right)$.

(f) It is immediate from Lemma $4.17(\mathrm{f})$ that $T_{1} \cdot T_{1} \cdot T_{2}=0$; hence, $T_{1} \cdot T_{2} \subseteq$ $Z^{\prime}$.

Lemma 4.20. Adopt the notation and hypotheses of Case 3B with $T_{2}^{2} \neq 0$. Let $K$ be any complete intersection ideal with $K \subseteq J$ and $J / K$ cyclic, $\mathbb{L}$ be the minimal resolution of $R /(K: J)$ which is shown in (2.1), and $p: L_{1} \rightarrow L_{2}$ be the map of (3.4). Then there exists an integer $b$, with $b \geq 6$, and there exists bases $e_{1}, \ldots, e_{n}$ for $L_{1} ; f_{1}, \ldots, f_{n-1}, f_{1}^{\prime}, \ldots, f_{n-1}^{\prime}$ for $L_{2} ; g_{1} \ldots, g_{n}$ for $L_{3}$; and $h$ for $L_{4}$ such that

(a) $K=\left(l_{1}\left(e_{1}\right), \ldots, l_{1}\left(e_{4}\right)\right)$,

(b) (2.3) holds,

(c) all products of basis vectors in $\bar{L}_{1} \cdot \bar{L}_{1}$ and $\bar{L}_{1} \cdot \bar{L}_{2}$ are zero except $e_{b} e_{i}=f_{i}$, $e_{i} f_{i}^{\prime} \equiv g_{b}$, and $e_{b} f_{i}^{\prime} \equiv-g_{i}$ for $1 \leq i \leq b-1$, and

(d) $p\left(e_{b}\right) \equiv f_{5}^{\prime}$ and $p\left(e_{i}\right) \equiv 0$ for all $i \neq 5$.

Proof. Let $h$ be any generator for $L_{4}$. We have two ways to view the multiplication in $T_{0}$. On the one hand, we can use the multiplication in $\overline{\mathbb{L}}$ to compute $T_{\bullet} \cdot T_{\bullet}$ as described in (4.1) and (4.2). On the other hand, Lemma 4.18 gives a complete description of all of the multiplication in $T_{\bullet}$, except the multiplication $T_{2} \cdot T_{2}$. In the present proof we use the interplay between these two descriptions of $T_{\bullet} \cdot T_{\bullet}$ in order to learn about the multiplication in $\overline{\mathbb{L}}$.

Let $e_{1}, \ldots, e_{4}$ be elements in $L_{1}$ with $\left(l_{1}\left(e_{1}\right), \ldots, l_{1}\left(e_{4}\right)\right)=K$. The hypothesis $t=4$, ensures that $e_{1}, \ldots, e_{4}$ is the beginning of a basis for $L_{1}$. Let $\varepsilon_{1}, \ldots, \varepsilon_{4}$ be the basis for $K_{1}$ which is defined by $\alpha_{1}\left(\varepsilon_{i}\right)=e_{i}$ for $1 \leq i \leq 4$, and let $x_{1}, \ldots, x_{5}$ be the basis for $T_{1}$ which is given above (4.6). According to Lemma 4.18, we may decompose $T_{2}$ into $k y \oplus Y$ with

$$
\operatorname{dim}\left(y \cdot T_{1}\right)=5 \text { and } T_{1} \cdot Y=0 .
$$

We know from Lemma 4.14(a) that $T_{1}^{2} \subseteq Y$; consequently,

$$
\left[\begin{array}{l}
\bar{\varphi} \\
0
\end{array}\right] \in Y \text { and }\left[\begin{array}{c}
0 \\
\bar{e}_{i}
\end{array}\right] \in Y
$$

for all $\varphi \in K_{2}$ and for all $i$ with $1 \leq i \leq 4$. It follows that we may modify $y$ in order to assume that

$$
y=\left[\begin{array}{c}
0 \\
\bar{e}_{0}
\end{array}\right]
$$


for some $e_{0} \in L_{1}$. It also follows that $L_{1}$ decomposes into $R e_{0} \oplus E$ where $\left(e_{1}, \ldots, e_{4}\right) \subseteq E$ and $E$ has the property that

$$
\left[\begin{array}{l}
0 \\
\bar{e}
\end{array}\right] \in Y
$$

for all $e \in E$. When the products of (4.21) are interpreted using (4.2), we see that $e_{0} e_{1}, \ldots, e_{0} e_{4}, p\left(e_{0}\right)$ is the beginning of a basis for $L_{2},\left(\bar{e}_{1}, \ldots, \bar{e}_{4}\right) \cdot \bar{E}=$ 0 , and $p(e) \equiv 0$ for all $e \in E$.

We next show that $E \cdot E \equiv 0$. We have observed that $\operatorname{dim} \bar{L}_{1}^{2} \geq 4$; consequently, a quick look at Theorem 2.2 shows that the multiplication in $\overline{\mathbb{L}}$ is given in multiplication table (c). In other words, there is a decomposition $\bar{L}_{1}=k v \oplus V$ with $V^{2}=0$. The fact that $\operatorname{dim} \bar{e}_{0} \cdot \bar{L}_{1} \geq 4$ ensures that $\bar{e}_{0} \notin V$; and therefore, $\bar{L}_{1}=k \bar{e}_{0} \oplus V$. It is easy to select a nonzero element $\bar{e}$ of $\left(\bar{e}_{1}, \ldots, \bar{e}_{4}\right) \cap V$. Indeed, if we write $\bar{e}_{i}=\lambda_{i} \bar{e}_{0}+v_{i}$ with $\lambda_{i} \in k$ and $v_{i} \in V$, then either $\lambda_{1}=0$ (in which case we take $\bar{e}=\bar{e}_{1}$ ) or $\lambda_{1} \neq 0$ (in which case we take $\left.\bar{e}=\lambda_{1} \bar{e}_{2}-\lambda_{2} \bar{e}_{1}\right)$. Let $\overline{e^{\prime}}=\lambda \bar{e}_{0}+v$ be an arbitrary element of $\bar{E}$. We know that $\left(\bar{e}_{1}, \ldots, \bar{e}_{4}\right) \cdot \bar{E}=0, V^{2}=0$ and $\operatorname{dim} \bar{e}_{0}\left(\bar{e}_{1}, \ldots, \bar{e}_{4}\right)=4$. It follows from

$$
0=\overline{e^{\prime}} \bar{e}=\left(\lambda \bar{e}_{0}+v\right) \bar{e}=\lambda \bar{e}_{0} \bar{e},
$$

that $\lambda=0$; thus $\bar{E} \subseteq V$ and $E \cdot E \equiv 0$.

We may decompose $E$ as ${ }^{\prime}\left(e_{1}, \ldots, e_{4}\right) \oplus E^{\prime} \oplus E^{\prime \prime}$, where

$$
\operatorname{dim} \bar{e}_{0}\left(\left(\bar{e}_{1}, \ldots, \bar{e}_{4}\right) \oplus \overline{E^{\prime}}\right)=\operatorname{dim}\left(\left(\bar{e}_{1}, \ldots, \bar{e}_{4}\right) \oplus \overline{E^{\prime}}\right)
$$

and $e_{0} E^{\prime \prime} \equiv 0$. Let $b-1$ denote the dimension of the vector spaces on line (4.22). Rename $e_{0}$ by calling it $e_{b}$. Pick any basis $e_{b+1}, \ldots, e_{n}$ for $E^{\prime \prime}$.

The hypothesis $T_{2}^{2} \neq 0$ guarantees that there are elements $v_{1}$ and $v_{1}^{\prime}$ in $L_{1}$ with $\bar{v}_{1} \bar{p}\left(\overline{v_{1}^{\prime}}\right) \neq 0$. We have seen that $\bar{L}_{1}=k \bar{e}_{b} \oplus \operatorname{ker} \bar{p}$; thus, $\bar{v}_{1} \bar{p}\left(\bar{e}_{b}\right)$ is a nonzero element of $\bar{L}_{3}$ for some $v_{1} \in L_{1}$. The multiplication $\bar{L}_{1} \otimes \bar{L}_{3} \rightarrow \bar{L}_{4}$ is a perfect pairing; consequently, $\bar{p}\left(\bar{e}_{b}\right) \cdot \bar{L}_{1}^{2} \neq 0$. On the other hand, we have seen that $\bar{L}_{1}^{2}=\bar{e}_{b} \bar{L}_{1}$. Thus, $\bar{p}\left(\bar{e}_{b}\right) \bar{e}_{b}$ is a nonzero element of $\bar{L}_{3}$. The very last assertion in Theorem 3.5 shows that $\bar{p}\left(\bar{e}_{b}\right) \bar{e}_{b}\left(\bar{e}_{1}, \ldots, \bar{e}_{4}\right)=0$. Thus, we may select a basis $e_{5}, \ldots, e_{b-1}$ for $E^{\prime}$ with $\bar{p}\left(\bar{e}_{b}\right) \bar{e}_{b} \bar{e}_{5}=\bar{h}$ and $\bar{p}\left(\bar{e}_{b}\right) \bar{e}_{b} \bar{e}_{i}=0$ for $6 \leq i \leq b-1$. Select the basis $g_{1}, \ldots, g_{n}$ for $L_{3}$ with the property $e_{i} g_{j}=\delta_{i j} h$. Observe that $\bar{p}\left(\bar{e}_{b}\right) \bar{e}_{b}=-\bar{g}_{5}$. Label $f_{i}=e_{b} e_{i}$ for $1 \leq i \leq b-1$ and $f_{5}^{\prime}=p\left(e_{b}\right)$ in $L_{2}$. Observe that $\left(f_{1}, \ldots, f_{b-1}\right)^{2}=0$ and $f_{i} f_{5}^{\prime} \equiv \delta_{i 5} h$ for $1 \leq i \leq b-1$. The proof of Lemma 2.6 (see [21] for details) allows us to extend $f_{1}, \ldots, f_{b-1}, f_{5}^{\prime}$ to be a basis $f_{1}, \ldots, f_{b-1}, f_{1}^{\prime}, \ldots, f_{b-1}^{\prime}$ of $L_{2}$ which satisfies (2.3). It is now clear that the basis we have constructed for $\mathbb{L}$ satisfies conditions (a)-(d).

Corollary 4.23. If the notation and hypotheses of Case 3B are adopted and $T_{2}^{2} \neq$ 0 , then $T_{\bullet}$ has the form $\mathbf{F}^{\star} \ltimes W$ for some trivial $\mathbf{F}^{\star}$-module $W$.

Proof. Let $K$ be any grade four complete intersection with $K \subseteq J$ and $J / K$ cyclic. Let $\mathbb{L}$ be the minimal resolution of $R /(K: J)$. Fix a basis for $\mathbb{L}$ as described in Lemma 4.20. Compute multiplication in $T_{\bullet}$ as described in (4.1) and (4.2). Consider the basis for $T_{\bullet}$ which is given in (4.6). We know from Lemma 4.14 that $\operatorname{dim} T_{1}^{2}=10$ and $T_{1}^{3}=0$; furthermore, the individual 
products in $T_{1}^{2}$ are given in (4.7). Use (4.2) and Lemma 4.20 to compute that all products in $T_{1} \cdot T_{2}$ are zero except

$$
x_{i} y_{b+6}=-z_{4+i} \text { for } 1 \leq i \leq 4 \text { and } \quad x_{5} y_{b+6}=z_{n+8} .
$$

The map $q: L_{2} \rightarrow L_{3}$ is defined below (3.4). It follows from Lemma 4.20(d) that $q\left(f_{5}\right) \equiv g_{b}$, but $q\left(f_{i}\right) \equiv q\left(f_{j}^{\prime}\right) \equiv 0$ for all $i \neq 5$ and all $j$. It is now clear that all products in $T_{1} \cdot T_{3}$ are zero except

$$
x_{i} z_{n+3+i}=-w_{b-4} \text { for } 1 \leq i \leq 4 \text { and } x_{5} z_{9}=-w_{b-4} .
$$

Finally, we use (4.2) and Lemma 4.20(d) once again to see that all products in $T_{2} \cdot T_{2}$ are zero except

$$
y_{11} y_{b+6}=w_{b-4} \text { and } y_{b+6} y_{b+6}=-2 w_{1} .
$$

Recall that 2 is a unit in $k$. There is no difficulty in verifying that $T_{\bullet}=\mathbf{F}^{\star} \ltimes W$ for some trivial $\mathbf{F}^{\star}$-module $W$.

The proof of Theorem 1.5 is complete.

\section{EXAMPLES AND QUESTIONS}

We begin this section by commenting on the hypotheses of Theorem 1.5. The hypothesis that $k$ have square roots is used only in the proof of Lemma 2.6 and it is not a particularly annoying hypothesis. Indeed, if $\left(R^{\prime}, \mathfrak{m}^{\prime}, k^{\prime}\right)$ is an arbitrary local ring, then the technique of residue field inflation (see, for example, [9, $\left.\left.0_{\mathrm{III}} 10.3 .1\right]\right)$ yields a faithfully flat extension $(R, \mathfrak{m}, k)$ of $R^{\prime}$ for which $k$ is closed under the square root operation. Many of the consequences of Theorem 1.5, applied to $R$, will descend back to $R^{\prime}$; however, we do not know if the conclusion of Theorem 1.5 will descend to $R^{\prime}$. The hypothesis that 2 is a unit in $R$ is also used only sporadically. There is a very trivial division by 2 at the end of the proof of Corollary 4.23; however, if the characteristic of $k$ had been two, then we would have calculated the second divided power $y^{(2)}$ of each element $y$ of $T_{2}$ and in particular, we would have written $y_{b+6}^{(2)}=-w_{1}$ instead of $y_{b+6}^{2}=-2 w_{1}$, thereby avoiding the division by 2 . The more serious use of $\operatorname{char} k \neq 2$ occurs when we appeal to Theorem 3.5. The proof of this result in [25] and [26] involves many divisions by 2 . We presume (but have not proved) that these divisions can be circumvented.

We next consider the question of the existence of grade four almost complete intersection ideals with predescribed Tor-algebras.

Question 5.1. Let $S_{\text {. }}$ be a graded $k$-algebra from the list in Theorem 1.5. Does there exist a grade four almost complete intersection ideal $J$ such that

$$
\operatorname{Tor}_{\bullet}^{R}(R / J, k) \cong S_{\bullet} \ltimes W
$$

for some trivial $S_{0}$-module $W$ ?

We are able to answer most of Question 5.1. All of the potential Tor-algebras which are listed in Theorem 2.2 for grade four Gorenstein ideals actually do exist (see [14] for Gorenstein rings whose Tor-algebras are described in Theorem 2.2(c)); consequently, the proof in Cases 1 and 2 (especially Table 4.13) can be read as an algorithm for producing an ideal $J$ for which (5.2) holds, provided $S_{\text {. }}$ is from the list $\mathbf{A}, \mathbf{B}[p], \mathbf{C}[p], \mathbf{C}^{(2)}, \mathbf{C}^{\star}, \mathbf{D}[p], \mathbf{D}^{(2)}, \mathbf{E}[p], \mathbf{E}^{(2)}$, and $\mathbf{E}^{(3)}$ 
with $0 \leq p$. In Examples 5.6, 5.7, and 5.8 we exhibit ideals $J$ for which (5.2) holds with $S_{\bullet}=\mathbf{F}[0], \mathbf{F}[1], \mathbf{F}[2], \mathbf{F}[3], \mathbf{F}^{(2)}, \mathbf{F}^{(4)}$, and $\mathbf{F}^{\star}$.

A more complete classification of Tor-algebras remains elusive.

Question 5.3. Let $S$. be a graded $k$-algebra from the list in Theorem 1.5. What are necessary and sufficient conditions on the vector space dimensions $\operatorname{dim} W_{i}$ in order that (5.2) hold with $W=\bigoplus_{i=0}^{4} W_{i}$ for some grade four almost complete intersection $J$ ?

For example, the proof of Theorem 1.5 shows that if $(5.2)$ holds with $S_{\bullet}=\mathbf{C}^{\star}$ for some grade four almost complete intersection $J$, then $W=0$. (In fact, the entire resolution of $R / J$ is known in this case.) On the other hand, every example that we have considered for which

$$
\operatorname{Tor}_{\bullet}^{R}(R / J, k) \cong \mathbf{F}^{\star} \ltimes W,
$$

also has $W=0$. We wonder if (5.4) implies that $W=0$; we also wonder if a structure theorem exists for the minimal resolution of $R / J$ for those $J$ which satisfy (5.4).

Finally, the variable of linkage class should also be thrown into the question about the classification of Tor-algebras. A number of years ago, Matthew Miller and the present author knew many Gorenstein rings of projective dimension four with $T_{1}^{2}=0$. None of these rings were in the linkage class of a complete intersection (licci). We conjectured that if $A$ is a licci Gorenstein ring of projective dimension four, then $T_{1}^{2} \neq 0$, and we deduced a number of consequences assuming that the conjecture held. Most of the consequences of the conjecture [20] have since been proved [11]; furthermore, various attempts to gather evidence for the conjecture have netted results which are interesting in their own right [22]. In the meantime, we have shown that the conjecture itself is false. The following question remains unanswered.

Question 5.5. Suppose $T_{\bullet}$ is the Tor-algebra of some Cohen-Macaulay ring. Does there exist a licci ring $A$ with $T_{\bullet}(A) \cong T_{\bullet}$ ?

Example 5.6. Let $Y_{1 \times 5}$ be a generic matrix, $X_{5 \times 5}$ be a generic alternating matrix, and $R$ be the local ring $k[X, Y]_{(X, Y)}$. Huneke and Ulrich [10, Proposition 5.8] introduced the grade four almost complete intersection $J=I_{1}(Y X)$. One can compute that $\operatorname{Tor}_{\bullet}^{R}(R / J, k)=\mathrm{F}^{\star}$. The Huneke-Ulrich almost complete intersection ideals are closely related to the Huneke-Ulrich deviation two Gorenstein ideals which have been studied rather extensively; see [15, 13, 28].

Example 5.7. Let $Y_{1 \times 4}$ and $X_{4 \times 3}$ be generic matrices and $v$ be an indeterminate. Consider the local ring $R=k[X, Y, v]_{(X, Y, v)}$. Let $I=\left(a_{1}, \ldots, a_{7}\right)$ be the grade four Gorenstein ideal with $a_{j}=\sum_{i=1}^{4} y_{i} x_{i j}$ for $1 \leq j \leq 3$ and $a_{4+j}=c_{j}+v y_{j}$ for $1 \leq j \leq 4$, where $c_{j}$ is equal to $(-1)^{j+1}$ times the determinant of $X$ with row $j$ removed. (The ideal $I$ is known as a Herzog ideal; see, for example, [19], [5, §3], or [23, Example 7.16].) If $J=\left(a_{1}, a_{4}, a_{5}, a_{6}\right): I$, then (5.2) holds with $S_{\bullet}=\mathbf{F}[2]$ and $W$ equal to $k(-2) \oplus k(-3)^{8} \oplus k(-4)$. If $J=\left(y_{1} a_{3}+a_{4}, a_{5}, a_{6}, a_{7}\right): I$, then (5.2) holds with $S_{\bullet}=\mathbf{F}[0]$ and $W=$ $k(-2)^{3} \oplus k(-3)^{12} \oplus k(-4)^{3}$.

Example 5.8. Let $I=\left(a_{1}, \ldots, a_{9}\right)$ be the grade four Gorenstein ideal defined in [18] with $\tau=5, x_{11}=1$, and $x_{21}=x_{31}=x_{41}=x_{51}=x_{12}=x_{13}=0$. If 


$$
\begin{aligned}
& J=\left(a_{i}, a_{j}, a_{k}, a_{l}\right): I, \text { then } \\
& \operatorname{Tor}_{\bullet}^{R}(R / J, k)=\mathbf{F}[1] \ltimes\left(k(-2)^{3} \oplus k(-3)^{12} \oplus k(-4)^{3}\right), \\
& \quad \text { if }\{i, j, k, l\}=\{3,5,6,7\}, \\
& =\mathbf{F}[3] \ltimes\left(k(-2)^{1} \oplus k(-3)^{8} \oplus k(-4)^{1}\right), \\
& \quad \text { if }\{i, j, k, l\}=\{1,5,6,7\}, \\
& =\mathbf{F}^{(2)} \ltimes\left(k(-2)^{3} \oplus k(-3)^{10} \oplus k(-4)^{3}\right), \\
& \quad \text { if }\{i, j, k, l\}=\{3,5,6,9\}, \\
& =\mathbf{F}^{(4)} \ltimes\left(k(-2)^{3} \oplus k(-3)^{6} \oplus k(-4)^{3}\right), \\
&
\end{aligned}
$$

\section{ACKNOWLEDGMENT}

A large number of examples were calculated using the "script" capability of version 3.0 of the MACAULAY program. The author sends his thanks to Bayer and Stillman for creating and sharing this wonderful program.

\section{REFERENCES}

1. E. Assmus, On the homology of local rings, Illinois J. Math. 3 (1959), 187-199.

2. L. Avramov, Homological asymptotics of modules over local rings, Commutative Algebra, Mathematical Sciences Research Institute Publications, no. 15, Springer-Verlag, Berlin, Heidelberg, New York, 1989, pp. 33-62.

3. Algebraic Geometry, papers from the conference held in Sundance, Utah, May 1990, (D. Eisenbud and C. Huneke, eds.), Jones and Bartlett, Boston, Mass., 1992.

4. L. Avramov and E. Golod, Homology algebra of the Koszul complex of a local Gorenstein ring, Mat. Zametki 9 (1971), 53-58; English transl., Math. Notes 9 (1971), 30-32.

5. L. Avramov, A. Kustin, and M. Miller, Poincaré series of modules over local rings of small embedding codepth or small linking number, J. Algebra 118 (1988), 162-204.

6. D. Buchsbaum and D. Eisenbud, Algebra structures for finite free resolutions, and some structure theorems for ideals of codimension 3, Amer. J. Math. 99 (1977), 447-485.

7. H. Charalambous, E. G. Evans, and M. Miller, Betti numbers for modules of finite length, Proc. Amer. Math. Soc. 109 (1990), 63-70.

8. D. Eisenbud, Homological algebra on a complete intersection, with an application to group representations, Trans. Amer. Math. Soc. 260 (1980), 35-64.

9. A. Grothendieck, Eléments de géométrie algébrique III, Inst. Hautes. Études Sci. Publ. Math. 11 (1961).

10. C. Huneke and B. Ulrich, Divisor class groups and deformations, Amer. J. Math. 107 (1985), 1265-1303.

11. _ The structure of linkage, Ann. of Math. (2) 126 (1987), 277-334.

12. C. Jacobsson, A. Kustin, and M. Miller, The Poincaré series of a codimension four Gorenstein ring is rational, J. Pure Appl. Algebra 38 (1985), 255-275.

13. S. Kim, Projective resolutions of generic order ideals, $\mathrm{Ph} . \mathrm{D}$. thesis, University of Illinois, Urbana, 1988.

14. A. Kustin, New examples of rigid Gorenstein unique factorization domains, Comm. Algebra 12 (1984), 2409-2439.

15. _ The minimal free resolutions of the Huneke-Ulrich deviation two Gorenstein ideals, J. Algebra 100 (1986), 265-304. 
16. __ Gorenstein algebras of codimension four and characteristic two, Comm. Algebra 15 (1987), 2417-2429.

17. A. Kustin and M. Miller, Algebra structures on minimal resolutions of Gorenstein rings of embedding codimension four, Math. Z. 173 (1980), 171-184.

18. _ Structure theory for a class of grade four Gorenstein ideals, Trans. Amer. Math. Soc. 270 (1982), 287-307.

19. _ Multiplicative structure on resolutions of algebras defined by Herzog ideals, J. London Math. Soc. (2) 28 (1983), 247-260.

20. ___ Tight double linkage of Gorenstein algebras, J. Algebra 95 (1985), 384-397.

21. _ Classification of the Tor-algebras of codimension four Gorenstein local rings, Math. Z. 190 (1985), 341-355.

22. A. Kustin, M. Miller, and B. Ulrich, Linkage theory for algebras with pure resolutions, J. Algebra 102 (1986), 199-228.

23. A. Kustin, M. Miller, and B. Ulrich, Generating a residual intersection, J. Algebra 146 (1992), 335-384.

24. M. Miller, Multiplicative structures on finite free resolutions, Free Resolutions in Commutative Algebra and Algebraic Geometry, papers from the conference held in Sundance, Utah, May 1990, (D. Eisenbud and C. Huneke, eds.), Jones and Bartlett, Boston, Mass., 1992.

25. S. Palmer, Algebra structures on resolutions of rings defined by grade four almost complete intersections, Ph.D. thesis, University of South Carolina, 1990.

26. __ Algebra structures on resolutions of rings defined by grade four almost complete intersections, J. Algebra (to appear).

27. J.-P. Serre, Sur la dimension homologique des anneaux et des modules noetheriens, Proc. Internat. Sympos. Algebraic Number Theory, 1955, Science Council of Japan, Tokyo, 1956, pp. 175-189.

28. H. Srinivasan, Minimal algebra resolutions for cyclic modules defined by Huneke-Ulrich ideals, J. Algebra 137 (1991), 433-472.

29. J. Tate, Homology of noetherian rings and local rings, Illinois J. Math. 1 (1957), 14-27.

30. J. Weyman, On the structure of free resolutions of length 3, J. Algebra 126 (1989), 1-33.

Department of Mathematics, University of South Carolina, Columbia, South CarOLINA 29208

E-mail address: kustin@milo.math.scarolina.edu 